\title{
VI. The impulsive motion of electrified systems
}

\section{G.F.C. Searle M.A. F.R.S.}

To cite this article: G.F.C. Searle M.A. F.R.S. (1907) VI. The impulsive motion of electrified systems, Philosophical Magazine Series 6, 13:73, 118-148, DOI: 10.1080/14786440709463588

To link to this article: http://dx.doi.org/10.1080/14786440709463588

$$
\text { 册 Published online: } 16 \text { Apr } 2009 .
$$

Submit your article to this journal $\pi$

LII Article views: 3

Q View related articles $\asymp$ 


\section{$\left[\begin{array}{ll}118 & ]\end{array}\right.$}

VI. The Imprelsire Motion of Electrified Systems. By G. F. C. Searle, M.A., H.R.S., University Lecturer it Experimental Plysies, Cambridge*.

\$1. WTHEN an electrified system is suddenly set into motion, a pulse of electromagnetic disturbance travels outwards from the system in all directions with the velocity of light, and this pulse carries energy and electromagnetic momentum away to infinity. In the following paper, a method is given of calculating the radiated energy and momentum for any given system, and the results are applied to a number of simple systems. The principles of electromagnetism are then applied to dednce the electric and the magnetic energies and the momenta of the systems, when they are in steady motion. Some of the results, as will be indicated in footnotes, have already been published by Dr. Oliver Heaviside and by Dr. M. Abraham, but others are, I believe, now given for the first time.

The method employed in calculating the electric and magnetic forces in the pulse is simply an extension to finite speeds of Prof. J. J. Thomson's investigation $\dagger$ of the pulse given off when the infinitesimal velocity of a charged sphere is suddenly destroyed.

$\S 2$. When a charged particle bas been at rest at a given point 0 for an infinite time, the lines of electric force are uniformly distributed round the particle. At the time $t=0$, let the particle be impulsively set into motion at a speed $u$, which is maintained constant in direction and magnitude. Then, at any subsequent time $t$, the field can be divided into two parts by a sphere of radius $v t$ described about $O$ as centre, $v$ being the velocity of light. The field outside this sphere is the same as if the particle had remained at rest, while the field inside the sphere is the same as if the particle had been in steady motion for an infinite time.

On the other hand, suppose that a charged particle, which has been moving along a straight line, with uniform velocity $u$, for an infinite time, is suddenly stopped, at $t=0$, as it reaches a given point $O$. Then, at any subsequent time $t$, a sphere of radius $v t$ will again divide the field into two parts. The field outside this sphere is the same as if the particle had continued in steady motion, while the field inside the sphere

* Cômmunicated by the Author.

+ Elements of the Mathematical Theory of Electricity and Magnetism. Thircl edition (1904), §287. 
is the same as if the particle had been at rest at 0 for an infinite time.

More generally, suppose that both the direction and the magnitude of the velocity are instantaneously changed so that the velocity of the particle is $u_{1}$ from $t=-\infty$ to $t=0$, and $u_{2}$ from $t=0$ onwards. Then, if a sphere of radius $v t$ be described abont 0 , the point where the impulse oceurs, the field outside the sphere is the same as if the particle had continued to move with velocity $u_{1}$, and the field inside the sphere is the same as if the particle had been in steady motion with velocity $u_{2}$ for an infinite time.

These results, which will be assumed in the present paper, can be established by the method of the " retarded potential" for points either inside or outside the sphere of radius $v t^{*}$. For external points they can also be established by Poisson's solution of the general equation $d^{2} \phi / d t^{2}=v^{2} \nabla^{2} \phi$.

$\S 3$. A point charge, $q$, in steady rectilinear motion, produces an electromagnetic field which travels with the charge. In this field the lines of electric force are straight and pass through $q$, while the lines of magnetic force are circles in planes normal to the direction of motion. If $\mathrm{R}$ and $\phi$, measured from $q$ as an origin and from the line of motion respectively, be the coordinates of any point, then, as was first shown by Dr. Oliver Heaviside $\dagger$, the electric force is given by

$$
\mathbf{E}=\frac{q\left(1-n^{2}\right)}{\bar{K}^{2}\left(1-n^{2} \sin ^{2} \phi\right)^{\frac{3}{2}}}, \quad . \quad . \quad .
$$

where $u$ is the velocity of the charge and

$$
n=u / v, \text {. . . . . . . }
$$

and $\mathrm{K}$ is the specific inductive capacity.

The magnetic force is given by

$$
\mathrm{H}=\mathrm{K} u \mathrm{E} \sin \phi . \quad \text {. . . . . . }
$$

The principles stated in $\& 2$, together with these values of $\mathrm{E}$ and $\mathrm{H}$, are sufficient for the solution of every problem connected with the motion of charged bodies.

* The most direct proof of the formulæ of the " retarded potential" is perhaps that given by Sommerfeld in his paper "Simplified deduction of the Field and the Forces of an Electron moving in any given way." Proceedings of the Koninklijke Academie van Wetenschappen to Amsterdam, Nov. 26, 1904.

+ 'The Electrician,' Dec. 7, 1888, p. 148, or ' Electrical Papers,' rol. ii. p. 511. 
Suppose that $O$ (fig. 1) is the position of $q$ at the time $t=0$ and $A$ its position at any later time $t$, the velocity between $O$ and $A$ being constant and equal to $u$. Let $\mathrm{P}$ be any point on the sphere of radius $r=v t$, described about $O$ as centre, and let the axis $O A$ cut the sphere in the pole $X$. Then $\mathrm{AP}=\mathrm{R}$ and $\mathrm{PAX}=\phi$, while $\mathrm{OA}=u t=n r$.

Let $\mathrm{POX}=\theta$. Then $r$ and $\theta$ are the coordinates of $\mathrm{P}$ relative to $O$, while $R$ and $\phi$ are its co-

Fig. 1.

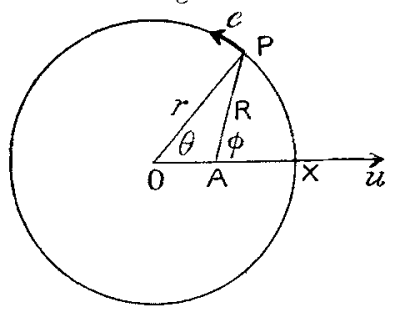
ordinates relative to $A$. If $\mathrm{E}_{\mathbb{N}}$ be the electric force at $\mathrm{P}$ along $O P$, we have, by (1),

But

$$
\mathrm{E}_{\mathrm{X}}=\frac{q\left(1-u^{2}\right) \mathrm{R} \cos (\phi-\theta)}{\mathrm{KR}^{3}\left(1-n^{2} \sin ^{2} \bar{\phi}\right)^{\frac{3}{2}}}
$$

$$
\begin{aligned}
\mathrm{R} \cos (\phi-\theta) & =\mathrm{OP}-\mathrm{OA} \cos \theta=r(1-n \cos \theta), \\
\mathrm{l} \sin \phi & =r \sin \theta,
\end{aligned}
$$

:Ind

$$
\mathrm{R}^{2}=r^{2}\left(1+n^{2}-2 n \cos \theta\right)
$$

Hence

Thus

$$
\mathrm{R}^{2}\left(1-n^{2} \sin ^{2} \phi\right)=r^{2}(1-n \cos \theta)^{2} .
$$

$$
\mathrm{K}_{\mathrm{N}}=\frac{q\left(1-n^{2}\right) r(1-n \cos \theta)}{\mathrm{K} r^{3}(1-n \cos \theta)^{3}}=\frac{q\left(1-u^{2}\right)}{\mathrm{K} r^{2}(1-n \cos \theta)^{2}} .
$$

If the particle was at rest at $\mathrm{O}$ until $t=0$, the electric force just outside the sphere $r=v t$ is radial to $O$ and normal to the sphere and has the value

$$
\mathrm{E}=q / \mathrm{K} r^{2} \text {. . . . . . . (5) }
$$

At the surface of the sphere there is therefore a discontinuity. in the normal component of the electric force, and, since there is no charge on the surface, this discontinuity can arise only from a flux of electric displacement along the surface. By symmetry this flux must be along lines of longitude.

Let $\mathrm{E}$ be the tangential electric force along the lines of longitude of the sphere $r=v t$ and let

$$
e=\int_{0}^{p} \mathrm{E} d z, \quad . \quad . \quad . \quad . \quad .
$$

where the integral is taken over the infinitesimal thickness, $p$, of the surface of the sphere, and $d z$ is an element of length measured along $p$. In other words, $\mathrm{K} e / 4 \pi$ is the surface tiux of 
electric displacement across unit length of a line of latitude. When $\mathrm{E}$ and $e$ are positive they are directed away from the pole X.

Now consider the spherical cap formed by the revolution of the are PX (fig. 1) about OX. The flux of displacement out from this cap by its edge and by its convex surface is

$$
(\mathrm{K} e / 4 \pi) .2 \pi r \sin \theta+\frac{1}{2} q(1-\cos \theta) .
$$

By (4) the flux of displacement into the cap by its concave surface is

or

$$
(\mathrm{K} / 1 \pi) \int_{0}^{\theta} \mathrm{E}_{\alpha} \cdot 2 \pi r^{2} \sin \theta d \theta,
$$

$$
\frac{1}{2} q\left(1-u^{2}\right) \int_{0}^{\theta} \frac{\sin \theta d \theta}{(1-n \cos \theta)^{2}}
$$

or

$$
\underline{\underline{2}} \frac{(1+n)(1-\cos \theta)}{2(1-n \cos \theta)} \text {. }
$$

Equating the inward to the outward flux, we find

and hence

$$
\text { Ker } \sin \theta=y\left\{\frac{1+n}{1-n \cos \theta}-1\right\}(1-\cos \theta),
$$

$$
e=\frac{q \sin \theta}{\mathrm{K} r(1-n \cos \theta)}=\frac{q u \sin \theta}{\mathrm{K} r(c-u \cos \theta)} . .
$$

This result is of great importance since all the formulæe for the radiation arising from the impulsive or gradual change of velocity of an electrified system can be deduced from it*.

Since $e$ is at right angles to the plane of $r$ and $u$ we can express it in the vector form

$$
\mathrm{e}=\frac{q}{\mathrm{~K} r} \frac{\operatorname{Vr}_{1} \operatorname{Vr}_{1} \mathrm{u}}{v-\mathrm{ur}_{1}}=\frac{q}{\mathrm{~K} r} \frac{\mathrm{r}_{1}\left(\mathrm{ur}_{1}\right)-\mathrm{u}}{v-\mathrm{ur}_{1}} . \quad .
$$

Here heavy type denotes vectors, and $r_{1}$ is a unit vector in the direction of $r$. Also $\mathbf{u r}_{1}$ is a scalar and $\operatorname{Vr}_{1} \mathbf{u}$ a vector product.

$\$ 4$. In the expanding spherical wave the disturbance is propagated at right angles to the electric force with velocity $i$, and hence the associated magnetic force, which is at right angles both to the electric force and to the direction of propagation, is given by the equation

$$
\mathrm{H}=\imath \mathrm{KE} \text {, }
$$

which holds in every case of propagation of electromagnetic waves.

* This result was first given by Dr. Heaviside, 'The Electrician,' Oct. 11,1901 . 
On the surface of the expanding sphere there will be a surface flux of magnetic induction along lines of latitude. If $\mu h$ be the flux per unit length of a line of longitude, we may deduce from the last equation that $h=r \mathrm{~K} e$. But it is more instructive to obtain this result in another way.

Take a fixed circle with its centre on the axis OX and its plane perpendicular to the axis, and let the circle subtend an angle $2 \theta$ at $O$ (fig. 1), the distance of $O$ from the circle being $r$. Then just before the expanding sphere reaches the circle the electric force is given by (5), while just after the sphere has passed over the circle, the electric force normal to the sphere is given by (4). Hence, by $\S 3$, the flux of electric displacement through the circle suddenly changes by the amount $\frac{1}{2} \mathrm{~K}_{e r} \sin \theta$. Thus, if $p$ be the infinitesimal thiekness of the wave, the time-integral of the line-integral of $\mathrm{H}$, round the circle, taken for the time $p / v$, is equal to $4 \pi$ times the change of flux of displacement, or in symbols

$$
2 \pi r \sin \theta \int_{0}^{p / v} \mathrm{H} d t=\frac{1}{2} \mathrm{~K} e r \sin \theta \cdot 4 \pi .
$$

Putting $d z=v d t$, we find that, if

then

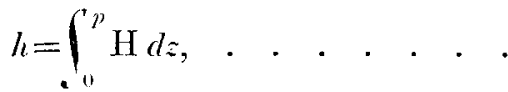

$$
\begin{aligned}
& h=r \mathbf{K} e \text {. }
\end{aligned}
$$

When $e$ is directed away from the pole $\mathrm{X}$, as in fig. 1, the directions of $h$ and of $u$ are connected in the same way as the rotation and translation of a right-handed screw worling in a fixed nut.

$\$ 5$. So far the charge has been supposed to be impulsively set in motion. If, however, it is originally in motion and is impulsively brought to rest, it is only necessary to take (4) as applying to the field outside the sphere and (5) as applying to the field inside it. Thus the directions of $e$ and $h$ are simply reversed, while their magnitudes remain unchanged.

$\$ 6$. When the charge $q$ is finite, there is a finite flux of displacement, viz. $K e / 4 \pi$, in the infinitely thin surface of the expanding spherical wave, and this implies an infinite value for $\mathrm{E}$ and involves an infinite amount of energy. But, as we shall see in $\S 7$, if a charge $Q$ is distributed over finite surfaces or throughout finite voiumes according to any given law of distribution, this difficalty disappears and the formula (7) enables us to calculate the electric and magnetic forces in the pulse due to the sudden starting or stopping of an electrified system when the pulse has travelled out a great distance from 
the system. The formula ( 7 ) is, indeed, applicable to the disturbance existing at any time after the impulsive change of velocity, but the necessary integrations are difficult unless we wail till the pulse has travelled out to a great distance.

$\$ 7$. If a sphere of radius $v t$ be described about every point on the surface of the system, in the position which it occupies at $t=0$, the wave disturbance at time $t$, due to an impulsive change of velocity of the system at $t=0$, will be confined to the region contained by the envelopes of these spheres. When it is very large compared with the linear dimensions of the system, this region will approximate to a spherical shell of variable thickness $p$, this thickness never exceeding the greatest distance between two points of the sxstem. When, as in the examples discussed in this paper, $p$ is infinitesimal compared with $v t$, the shell may be constructed in the mamner indicated in fig. 2. Any point 0 , within or

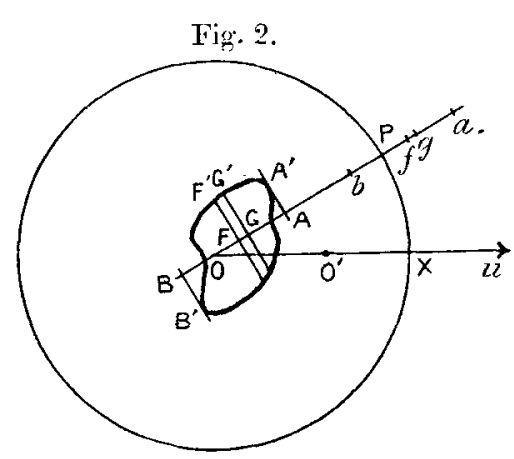
very close to the system, is chosen as origin and a sphere of radius $v t$ is described about it as centre, and $P$ denotes any point on this sphere. Two planes, $\mathrm{AA}^{\prime}$ and $\mathrm{BB}^{\prime}$, normal to $\mathrm{OP}$, are drawn touching the system at $A^{\prime}$ and $B^{\prime}$ and cutting $O P$ in $A$ and $B$, the plane $\mathrm{AA}^{\prime}$ being the nearer to $P$. If more than two tangent planes can be drawn, we must select the one that is nearest to $P$ and the one that is farthest from $P$. If, now, on the radius $O P$ we take $\mathrm{P} a=\mathrm{OA}$ and $\mathrm{P} b=\mathrm{OB}$, then $p$, the thickness of the shell or pulse in the neighbourhood of $\mathrm{P}$, will be equal to $a b$.

Let $O X$, drawn in the direction of $n$, cut the sphere in $X$, and let $\mathrm{OX}=\mathrm{OP}=r$ and $\mathrm{POX}=\theta$. Let $\mathrm{O}^{\prime}$ be a point on OX at a distance $u t$ or $n r$ from $\mathrm{O}$.

Now take a point $f$ on OP between $a$ and $b$ at a distance $z$ from $\mathrm{P}$ and draw a plane $\mathrm{FF}^{\prime}$ normal to $\mathrm{OP}$ and at a distance $v t$ from $f$, so that it intersects the system and cuts $\mathrm{OP}$ in $\mathrm{F}$. Then, in the limit, any point of the section of the system by this plane may be considered as being at a distance $v t$ from $f$ and, further, the straight line joining it to $\mathrm{P}$ may be treated as being parallel to $O P$. To this approximation, the wave disturbance at $f$ at time $t$ is due entirely to the charged particles which lie in the plane $\mathrm{FF}^{\prime}$, and the effect of these particles is the same as if they were concentrated at the point $F$. 
In practical cases, however, the electrification is not concentrated in ideal poini-charges, but is spread over surfaces or distributed through volumes. We therefore take a second plane $\mathrm{GG}^{\prime}$, parallel to $\mathrm{FF}^{\prime}$ and at a distance $d z$ from it, and a corresponding point $g$ on $\mathrm{OP}$. Then, at the time $t$, the only points on $\mathrm{OP}$, which experience any wave disturbance from the electrification $d \mathrm{Q}$ contained between the planes $\mathrm{FF}^{\prime}$ and $\mathrm{GG}^{\prime}$, are the points between $f$ and $g$, and, further, the wave clisturbance at points between $f$ and $g$ at time $t$ is entirely due to the electrification $d Q$. It is clear that, in the limit, the electric force in the pulse at any point between $f$ and $g$ may be calculated by substituting $d \mathrm{Q}$ for $q$ in (7) and then supposing that de, the corresponding total flux of electric displacement, no longer takes place along a mathematical surface, but is uniformly distributed in a layer of thickness dz. We thus obtain for the tangential electric force at any point of the pulse

$$
\mathrm{E}=\frac{u \sin \theta}{\mathrm{K} r(v-u \cos \theta)} \frac{d \mathrm{Q}}{d z} . \quad . \quad . \quad .
$$

In the same way, the corresponding total flux of magnetic induction, $\mu d h$, is uniformly distributed through a layer of thickness $d z$, and thus, by (10), the magnetic force in the pulse is given by

$$
\mathrm{H}=v \mathrm{KE}=\frac{u v \sin \theta}{r(v-u \cos \theta) d \mathrm{Q}} \ldots . \quad, \quad .
$$

Since $\mu \mathrm{K} v^{2}=1$, it follows that, at any point of the pulse, $\mu \mathrm{H}^{2} / 8 \pi=\mu v^{2} \mathrm{~K}^{2} \mathrm{E}^{2} / 8 \pi=\mathrm{KE}^{2} / 8 \pi$, and hence the magnetic energy per unit volume is equal to the electric energy per unit volume. Thus, when $d \mathrm{Q} / d z$ is known in terms of the angular coordinates of $\mathrm{P}$, we can calculate the energy in the pulse due to the impulsive change in the velocity of the system.

$\S 8$. Within the region of wave disturbance there is a gradual transition of the field just outside the expanding pulse into the field just inside the pulse. In each of these fields the electric force ultimately varies as $1 / r^{2}$, whereas the tangential electric force, arising from the sudden change in the velocity of the system, ultimately varies as $1 / r$. Hence, when $r$ becomes very great, the only electric force which need be considered is the tangential electric force $\mathrm{E}$, given by (11).

If we wish to do so, we can easily calculate the actual electric and magnetic forces at any point $f$ (fig. 2) within the pulse at any great time after the body has been set into 
motion. For the pulse due to the part of the system which lies between $f$ and the corresponding plane $\mathrm{FF}^{\prime}$ has already passed over $f$, while the pulse due to the remainder of the system has not yet reached $f$. If $Q_{1}$ is the charge between $f^{\prime}$ and the plane $\mathrm{FF}^{\prime}$ and $Q_{2}$ is the remainder of the total charge, and if $\mathrm{E}_{1}$ and $\mathrm{E}_{2}$ are the electric forces due to $\mathrm{Q}_{1}$ and $\mathrm{Q}_{2}$, then the electric force at $f$ has the three components $\mathrm{E}_{1}$, $\mathrm{E}_{2}$, and $\mathrm{E}$, the latter being giren by (11). The electric force $\mathrm{E}_{2}$ has the value $\mathrm{Q}_{2} / \mathrm{K} r^{2}$ and is ultimately radial to $\mathrm{O}$, but the force $\mathrm{E}_{1}$ is ultimately radial to $\mathrm{O}^{\prime}$ (fig. 2 ) and has the value

$$
\mathrm{E}_{1}=\frac{\mathrm{Q}_{1}\left(1-n^{2}\right)}{\mathrm{KR}^{2}\left(1-n^{2} \sin ^{2} \phi\right)^{3}},
$$

where $R=O^{\prime} P$ and $\phi$ is the angle between $O^{\prime} P$ and $O X$.

The magnetic force at $f$ has the three components $\mathrm{H}_{1}, \mathrm{H}_{2}$, and $\mathrm{H}$, where $\mathrm{H}$ is given by (12) and

$$
\mathrm{H}_{1}=\mathrm{K} u \mathrm{E}_{1} \sin \phi, \quad \mathrm{H}_{2}=0 .
$$

Both $\mathrm{H}_{1}$ and $\mathrm{HI}$ are in circles in planes normal to OX.

$\$ 9$. The electric and magnetic forces in the pulse ultimately vary as $1 / r$, while the thickness of the pulse, for given angular coordinates of $\mathrm{P}$, ultimately becomes constant, and hence the electromagnetic energy in the pulse tends to a constant value, $\mathrm{W}$, as $v t$, the radius of the pulse, becomes infinite. Since only the squares of $\mathrm{E}$ and $\mathrm{H}$ are concerned, it follows that the energy in the pulse is ultimately the same, whether the system is suddenly set into motion at speed $u$ or is suddenly reduced to rest from speed $u$, the direction of $u$ relative to the system being the same in both cases. The energy $W$, which is radiated away in the pulse, cannot be recovered and may therefore be spoken of as " lost."

$\S 10$. We now proceed to show that the total energy of the system, for steady motion at speed $u$, is simply equal to $\mathrm{W}+\mathrm{U}_{0}$, where $\mathrm{U}_{0}$ is the electrostatic energy of the system at rest. The total energy at the speed $u$ is $\mathrm{U}+\mathrm{T}$, the sum of the electric and magnetic energies, where

$$
\mathrm{U}=\frac{\mathrm{K}}{8 \pi} \iiint_{-} \mathrm{E}^{2} d x d y d z, \quad \mathrm{~T}=\frac{\mu}{8 \pi} \iiint_{\mathrm{H}^{2}} d \imath d y d z .
$$

If the moving system be brought to rest suddenly, the charges do not move under the influence of the electric forces, which act on them after the system has been brought to rest, and therefore the electromagnetic field does no work upon the system after its motion is destroyed*. Hence the total

* See M. Abrahan, Theorie der Elektrizitit, vol. ii. p. 280. 
energy in the field is unaltered by the sudden stopping of the system.

After a short time the pulse will get clear of the system and then it separates the "old field," which is external to the onter surface of the pulse, from the "new field," which is internal to the inner surface of the pulse. In the old field the electric and magnetic forces are those due to the undisturbed steady motion of the system, while in the new field the forces are those due to the system at rest. As the pulse advances, the energy in the old field continually diminishes to zero, the energy in the new field continually increases to $\mathrm{U}_{0}$, and the energy in the pulse tends to the value $W$. But the energy in the whole field has not been changed by stopping the system, and thus

$$
\mathrm{U}+\mathrm{T}=\mathrm{W}+\mathrm{U}_{0} . \ldots . \quad . \quad .
$$

The force required to keep the system at rest does not necessarily vanish directly the velocity of the system has been destroyed, but only after the pulse has got clear of the system. Thus, in the case of a charged sphere of radius a the time $2 a / v$ will elapse before the pulse gets clear of the sphere. After this time the sphere experiences no force.

$\S 11$. When the system is impulsively set into motion at speed $u$, a force may be required to maintain the speed at the constant value $u$ until the pulse gets clear of the system. As long as the force is necessary, it will do work in causing the charges on the system to move while they are under the influence of the electric forces in the pulse*, and thus the action of the force changes the energy of the electromagnetic field. When a sphere of radius $a$ is set into motion, the time $2 a /(v-u)$ will elapse before the pulse gets clear of the sphere. After this time no force is required to keep the velocity of the sphere constant.

As soon as the pulse bas got clear of the system, the energy of the electromagnetic field remains unchanged. In the old field, which is external to the outer surface of the pulse, the electric and magnetic forces are those due to the system at rest. As the pulse advances, the energy in the old field continually diminishes to zero, the energy in the new field continually increases to $\mathrm{U}+\mathrm{T}$, and the energy in the pulse tends to W. Hence, ultimately, the energy in the field has been increased from $\mathrm{U}_{0}$ to $\mathrm{U}+\mathrm{T}$, while $\mathrm{W}$ has been lost by radiation. Hence, in setting the system in motion, the force

* Since $\mathbf{H}$ is perpendicular to $u$, the force on a unit moving charge due to $\mathrm{H}$ is $\mu u \mathrm{H}$ at right angles to $u$. Hence no work is done in moving the charge while it is under the induence of this force. 
does the work $\mathrm{U}+\mathrm{T}+\mathrm{W}-\mathrm{U}_{0}$, and this, by (13), is equal to $2 W$. Thus, when a system is set into motion, half the work done by the applied force goes to increase the energy of the field, while the other half is lost by radiation.

$\$ 12$. It can be shown that the force exerted on any system of charges by the electromagnetic field together with the force

$$
\mathbf{G}=\frac{\mu \mathrm{K}}{4 \pi} \frac{d}{d t} \iiint \mathrm{V} \mathbf{E H} d x d y d z,
$$

where the integral is taken throughout the space within any surface $S$ which encloses the system, is equivalent to the resultant of the Maxwell stress over $\$$. Here, and throughout the paper, heavy type signities vectors and VEH denotes the vector product of $\mathbf{E}$ and $\mathbf{H}$. The differentiation with regard to the time is to be performed on the values of $\mathbf{E}$ and $\mathbf{H}$ at points fixed in space.

When a system, which has been at rest from $t=-\infty$ to $t=0$, is made to move in any given manner, from $t=0$ onwards, by the application of a set of mechanically applied forces, we can take the surface $S$ so great that, at any time $t$, the field at every point of $S$ is the old statical field due to the system at rest, and then the resultant of the Maxwell stress over $\mathrm{S}$ vanishes *. In this case, the resultant force exerted by the electromagnetic field upon the system is $-G$ and hence, if the system is to move in the given manner, there must be applied to the system by non-electromagnetic means, in addition to any force required to change the momentum of ordinary matter, a resultant force $F$, where $\mathbf{F}=\mathbf{G}$. Hence we find that, to produce the given motion, we require a force $\mathbf{F}$, where

$$
\mathbf{F}=\frac{\mu \mathrm{K}}{4 \pi} \frac{d}{d t} \iiint \mathrm{VEH} d x d y d z, \quad . \quad . \quad .
$$

in addition to any force required for the motion of ordinary matter.

The force $F$ is measured, on Newton's system, by the rate at which it could generate momentum in ordinary matter. Hence we may speak of $\boldsymbol{F}$ as generating momentum in the electromagnetic field and may say that the expression

$$
{ }_{4 \pi}^{\mu \mathrm{K}} \iiint_{d} \mathrm{VEH} d x d y d z \quad . \quad . \quad .
$$

is the momentum of the electromagnetic field.

* It is here supposed that there is no other system within a finite distance of the one under consideration. 
We are thus led to regard the momentum as being distributed throughout the field. If $\mathbf{m}$ denote the amount $\mathrm{per}^{\circ}$ unit volume,

$$
\mathrm{m}=(\mu \mathrm{K} / 4 \pi) \mathrm{VEH} . . . \quad \text {. . . }
$$

If $\mathbf{I}=\int \mathbf{F} d t$, so that $\mathbf{I}$ is the "impulse" of the applied force,

$$
\mathrm{I}=\frac{\mu \mathrm{K}}{4 \pi} \iiint \mathrm{VEH} d x d y d z . . .
$$

$\S 13$. When the system is set into steady motion from rest, the work done by the applied force $\mathbf{F}$ is $\int \mathbf{F u} d t$, where $\mathbf{F u}$ denotes the scalar product of $\mathbf{F}$ and $\boldsymbol{u}$. We have alreadyfound that this work is $2 \mathrm{~W}$, and thus

$$
\int \mathrm{Fu} d t=2 \mathrm{~W} \text {. . . . . . . }
$$

Since $\mathfrak{u}$ is constant, we have

$$
\mathrm{uI}=2 \mathrm{~W}=2\left(\mathrm{~T}+\mathrm{U}-\mathrm{U}_{0}\right) . . \quad . \quad .
$$

This result is applicable whatever the form of the system; it is not restricted to systems of revolution.

As soon as the pulse bas got clear of the system, the force $F$ vanishes and the total momentum in the field preserves a steady value. By $\$ 9$ we see that the momentum in the pulse tends to a constant value, which we denote by $\mathbf{P}$, as $t$, the radius of the pulse, becomes infinite. The momentum in the new field, which is enclosed by the inner surface of the pulse, also tends, therefore, to a constant value $\mathbf{M}$, which tho system has when it is in steady motion. Hence

$$
\mathbf{I}=\mathbf{M}+\mathbf{P} . \quad \cdot \quad \cdot \quad \cdot \quad \text {. }
$$

$\$ 14$. In certain cases we can express $\mathbf{M}$, the momentum of the field, for the system in steady motion, in terms of ' $\mathrm{T}$, the magnetic energy for steady motion. For steady motion we have, as in (3),

$$
\mathrm{H}=\mathrm{KVuE}, \text {. . . . . . }
$$

and hence, by (16),

$$
\begin{aligned}
\mathrm{m} & =(\mu \mathrm{K} / 4 \pi) \mathrm{VEH} \\
& =\left(\mu \mathrm{K}^{2} / 4 \pi\right) \mathrm{VE} \mathrm{VuE} \\
& =\left(\mu \mathrm{K}^{2} / 4 \pi\right)\left(\mathbf{u} . \mathrm{E}^{2}-\mathrm{E} . \mathrm{Eu}\right) . \quad . \quad . \quad .
\end{aligned}
$$

Now let $\mathrm{E}_{1}$ be the component of $\mathrm{E}$ parallel to $u$ and $\mathrm{E}_{2}$ and $\mathrm{E}_{3}$ the other rectangular components. Then $\mathrm{Eu}=\mathrm{E}_{1} u$ and 
$(\mathrm{VuE})^{2}=u^{2}\left(\mathrm{E}_{2}^{2}+\mathrm{E}_{3}^{2}\right)$, and hence

$$
\mathrm{H}^{2}=\mathrm{K}^{2} u^{2}\left(\mathrm{E}_{2}{ }^{2}+\mathrm{E}_{3}^{2}\right)
$$

But, by (22), the component of $\mathrm{m}$ parallel to $\mathrm{u}$ is

and hence

$$
\begin{array}{r}
m_{1}=\left(\mu \mathrm{K}^{2} / 4 \pi\right)\left(\mathrm{E}^{2}-\mathrm{E}_{1}{ }^{2}\right) u=\mu \mathrm{H}^{2} / 4 \pi u, . \\
\mathrm{M}_{1}=2 \mathrm{~T} / u \text {. . . . . . . }
\end{array}
$$

The other components of $m$ have the values

$$
m_{2}=-\left(\mu \mathrm{K}^{2} / 4 \pi\right) \mathrm{E}_{2} \mathrm{E}_{1} u, \quad m_{3}=-\left(\mu \mathrm{K}^{2} / 4 \pi\right) \mathrm{E}_{3} \mathrm{E}_{1} u \text {. }
$$

In special eases, as when the system has an axis of symmetry parallel to $u$, the components $\mathrm{M}_{2}$ and $\mathrm{M}_{3}$ will vanish, and then we have simply

$$
\mathrm{M}=2 \mathrm{~T} / u \text {. }
$$

$\S 1 \tilde{s}$. When $\mathbf{M}$ and $\mathbf{I}$ are both parallel to $u$, we can at once write down $\mathbf{P}$, the momentum in the pulse. For, by (20), $\mathbf{P}=\mathbf{I}-\mathbf{M}$, and hence, in this case, by (19) and (27),

$$
\mathrm{P}=2 \mathrm{~W} / u-2 \mathrm{~T} / u \text {. . . . . }
$$

Thus, by (13),

$$
\mathrm{P}=2\left(\mathrm{U}-\mathrm{U}_{0}\right) / u \text {. . . . . . }
$$

If we can calculate $P$ and $W$ for the pulse, we can deduce from them not only the electric and the magnetic energies of the system in steady motion but its momentum as well. For, by (29), (28), and (27),

$$
\begin{aligned}
\mathrm{U} & =\frac{1}{2} u \mathrm{P}+\mathrm{U}_{0} . \\
\mathrm{T} & =\mathrm{W}-\frac{1}{2} u \mathrm{P} . \\
\mathrm{M} & =2 \mathrm{~W} / u-\mathrm{P} .
\end{aligned}
$$

$\S 16$. The impulse of the force required to suddenly stop a system, which is moving along an axis of symmetry, is easily found. For since both $\mathrm{E}$ and $\mathrm{H}$ in the pulse change signs, when the system is stopped instend of being started, we see that the momentum in the pulse is the same in both cases. Hence, before the stopping, the momentum is M, while at a great time after the stopping the momentum is $\mathbf{P}$, since the Maxwell stress over an infinite surface enclosing the pulse vanishes, because $\mathrm{E}$ and $\mathrm{H}$ at points outside the pulse ultimately vary as $1 / r^{2}$. Hence, if $I^{\prime}$ be the impulse, in the opposite direction to $u$, which is required to reduce the system to rest,

Phil. Mag. S. 6. Vol. 13. No. T3. Jan. 1907. 
Hence the impulse required to start the system exceeds that required to stop it by twice the momentum carried away by the pulse in either case.

$\S 17$. We will now apply the principles explained in the earlier: part of this paper to some simple systems, and will calculate the energy and momentum in the pulse formed when the system is set into motion at speed $u$. By $\S 7$, the electric and magnetic energies in the pulse are equal, and thas the total energy in the pulse is ultimately equal to

$$
W=\frac{\mu}{4 \pi} \int \mathrm{H}^{2} r^{2} d \omega d z
$$

where $d \omega$ is an element of solid angle. Hence, by (11), since $u / v=n$, we have

$$
W=\frac{\mu u^{2}}{4 \pi} \int \frac{\sin ^{2} \theta d \omega d z}{(1-n \cos \theta)^{2}}\left(\frac{d Q}{d z}\right)^{2} \ldots .
$$

In the pulse, $\mathrm{E}$ and $\mathrm{H}$ are perpendicular to the radius $r$ and to each other. Hence, attending to the directions of $\mathbf{E}$ and $\mathbf{H}$, we see that VEH is in the direction of the outward radius and that its magnitude is simply $\mathrm{EH}$. Hence, by $(16)$, if $\mathbf{r}_{1}$ denote a unit vector in the direction of the radius, the momentum in the pulse is given, in vector notation, by

$\mathbf{P}=\frac{\mu \mathrm{K}}{4 \pi} \iint \mathbf{r}_{1} \mathrm{EH} r^{2} d \omega d z=\frac{\mu u n}{4 \pi} \iint \frac{\mathbf{r}_{1} \sin ^{2} \theta d \omega d z}{(1-n \cos \theta)^{2}}\left(\frac{d \mathrm{Q}}{d z}\right)^{2} \cdot$

Here and in (34) the range of $z$ is from $p_{1}$ to $p_{2}$, where $p_{2}-p_{1}=p$, the thickness of the pulse.

The component of $\mathbf{r}_{1}$ in the direction of $\mathfrak{u}$ is $\cos \theta$, and hence when, for any reason, $\mathbf{P}$ is parallel to $\mathfrak{u}$, it has the value

$$
\mathrm{P}=\frac{\mu n n}{4 \pi} \iint \frac{\sin ^{2} \theta \cos \theta d \omega d z}{(1-n \cos \theta)^{2}}\left(\frac{d \mathrm{Q}}{d z}\right)^{2} \ldots .
$$

When the system is symmetrical round an axis parallel to the direction of motion, we may put $d \omega=2 \pi \sin \theta d \theta$, and then

$$
\begin{aligned}
& \mathrm{W}=\frac{\mu u^{2}}{2} \iint \frac{\sin ^{3} \theta d \theta d z}{(1-n \cos \theta)^{2}}\left(\frac{d \mathrm{Q}}{d z^{2}}\right)^{2} . \\
& \mathrm{P}=\frac{\mu u n}{2} \iint \frac{\sin ^{3} \theta \cos \theta d \theta d z}{(1-n \cos \theta)^{2}}\left(\frac{d \mathrm{Q}}{d z}\right)^{2} .
\end{aligned}
$$

where $\theta$ goos from 0 to $\pi$.

$\S 18$. Suall Velocities.-Let the radius be defined by $\theta$ 
and $\phi$, where $\phi$ is measured round the axis of motion. Then

$$
\int\left(\frac{d \mathrm{Q}}{d z}\right)^{2} d z
$$

has the same value for the radius defined by $\pi-\theta$ and $\phi+\pi$ as for the radius defined by $\theta$ and $\phi$. Hence, if we expand $(1-n \cos \theta)^{-2}$ in the expression (35) for $\mathbf{P}$ and integrate term by term, we see that the integral of the first term is zero since, for a given value of $d w$, the parts of the pulse defined by $\theta$ and $\phi$ and by $\pi-\theta$ and $\phi+\pi$ make equal and opposite contributions to that term. Thus, going as far as the second term, we find that for small values of $n$

$$
\mathbf{P}=\frac{\mu u n n^{2}}{2 \pi} \iint_{0}\left(\frac{d \mathrm{Q}}{d z}\right)^{2} \mathrm{r}_{1} \sin ^{2} \theta \cos ^{2} \theta d \omega d z .
$$

Hence, for small speeds, $\mathbf{P}$ is proportional to $n^{3}$, and therefore, by (29), $\mathrm{U}-\mathrm{U}_{0}$ is proportional to $n^{4}$. By (13) it follows that $\mathrm{W}-\mathrm{T}$ is proportional to $n^{4}$.

\$19. Sphere with a uniform surface-charge.-This is, so far as I know, the only system to which the method explained in the present paper has been applied. The application was made by Dr. Heaviside*. If $Q$ be the charge and $a$ the radius of the sphere, $d \mathrm{Q} / d z=\mathrm{Q} / 2 a$, so that $d \mathrm{Q} / d z$ is independent of $z$, and thus the integrations with respect to $z$ can be made at once. We thus obtain, by (37),

$$
\mathrm{W}=\frac{\mu \mathrm{Q}^{2} u^{2}}{4 \iota} \int_{0}^{\pi} \frac{\sin ^{2} \theta d \theta}{(1-n \cos \theta)^{2}},
$$

where $n=u / v$. Putting $1-n \cos \theta=h$, we find

$$
\begin{aligned}
& W=\begin{array}{c}
\mu Q^{2} n^{2} \\
4 u n
\end{array} \int_{1-n}^{1+n}\left(\frac{n^{2}-1}{n^{2} / l^{2}}+\frac{2}{n^{2} / h}-\frac{1}{n^{2}}\right) d l, \\
& =\frac{\mu \cdot Q^{2} v^{\circ}}{2 a}\left(\begin{array}{l}
v \\
n
\end{array} \log \frac{v+u}{v-u}-2\right), \\
& =\frac{\mu \mathrm{Q}^{2} u^{2}}{a}\left(\frac{1}{3}+\frac{u^{2}}{j u^{2}}+\frac{u^{+}}{7 c^{4}}+\ldots\right) .
\end{aligned}
$$

The electrostatic energy of the sphere at rest is given by

$$
\mathrm{U}_{0}=\frac{\mathrm{Q}^{2}}{2 \mathrm{~K}^{2} t}=\frac{\mu t^{2} \mathrm{Q}^{2}}{2 a} \text {. }
$$

* 'The Electrician,' Nov. 29, 1901, p. 210.

K 2 
Thus since, by (13), U+T, the total energy of the sphere in steady motion, is $\mathrm{W}+\mathrm{U}_{0}$, we have

$$
\mathrm{U}+\mathrm{T}=\frac{\mu v^{2} \mathrm{Q}^{2}}{2 a}\left(\frac{v}{u} \log \frac{v+u}{v-u}-1\right)=\frac{\mathrm{Q}^{2}}{2 \mathrm{~K} a}\left(\frac{v}{u} \log \frac{v+u}{v-u}-1\right) .
$$

This value is identical with that which $I$ found* by integration throughout the whole field of the sphere in steady motion.

Since the sphere moves along an axis of symmetry, the momentum carried off in the pulse is, by (38),

$$
\mathrm{P}=\frac{\mu u n \mathrm{Q}^{2}}{4 \iota} \int_{0}^{\pi} \frac{\sin ^{3} \theta \cos \theta d \theta}{(1-n \cos \theta)^{2}} .
$$

Putting $1-n \cos \theta=h$ and using $\mu \mathrm{K} v^{2}=1$, we find

$$
\begin{aligned}
\mathrm{P} & =\frac{\mathrm{Q}^{2}}{4 a n u \mathrm{~K}} \int_{1-n}^{1+n}\left\{\frac{n^{2}-1}{h^{2}}+\frac{3-n^{2}}{h}-3+h\right\} d h, \\
& =\frac{\mathrm{Q}^{2} v}{4 a u^{2} \mathrm{~K}}\left\{\left(3-\frac{u^{2}}{r^{2}}\right) \log \frac{v+u}{v-u}-6 \frac{u}{v}\right\}, \\
& =\frac{\mathrm{Q}^{2} u^{3}}{a \mathrm{~K} v^{4}}\left(\frac{2}{3.5}+\frac{4 u^{2}}{5.7 v^{2}}+\frac{6 u^{4}}{7.9 v^{4}} \ldots\right) .
\end{aligned}
$$

Hence, as was proved in $\S 18$, for small values of $u / v$ the momentum carried off by the pulse varies as $u^{3}$.

From the values of $\mathrm{W}$ and $\mathrm{P}$ those of $\mathrm{U}, \mathrm{T}$, and $\mathrm{M}$ can be deduced by the method explained in $\$ 15$.

$\S 20$. Sphere with a uniform volume-charge.-If $\approx$ be measured from the centre of the sphere, we have, in this case,

$$
\frac{d \mathrm{Q}}{d z}=\frac{3 \mathrm{Q}\left(a^{2}-z^{2}\right)}{4 a^{3}}
$$

Hence

and thus, by (37),

$$
\int_{-a}^{+a}\left(\frac{d \mathrm{Q}}{d z}\right)^{2} d z=\frac{3 \mathrm{Q}^{2}}{5}
$$

$$
\mathrm{W}=\frac{\mu \mathrm{Q}^{2} u^{2}}{4 a} \cdot \frac{6}{5} \int_{0}^{\pi} \frac{\sin ^{3} \theta d \theta}{(1-n \cos \theta)^{2}}
$$

and hence, by $\S 19$,

$$
\mathrm{W}=\frac{6}{5} \cdot \frac{\mu r^{2} \mathrm{Q}^{2}}{2 a}\left(\frac{v}{u} \log \frac{v+u}{v-u}-2\right) .
$$

* Proc. Royal Soc. vol. lix. p. 344 (1896), and Phil. Irag. Oct. 1897, p. 340 . 
Thus the energy carried off in the pulse in the case of the volume-charge is six fifths of the energy carried off in the case of a surface-charge.

In exactly the same way, it follows that $P$ for a sphere with a volume-charge is six fifths of the value for a sphere with an equal surface-charge.

The electrostatic potential at a point within the sphere at a distance $r$ from its centre, when the sphere is at rest, is easily found to be

$$
\mathrm{Q}\left(3 a^{2}-r^{2}\right) / 2 \mathrm{~K} a^{3}
$$

and hence by the formula

$$
\mathrm{U}_{0}=\frac{1}{2} \Sigma \text { charge } \times \text { potential, }
$$

the electric energy is given by

$$
\mathrm{U}_{0}^{-}=\frac{3 \mathrm{Q}^{2}}{5} \frac{\overline{\mathrm{K}} a}{\dot{\mathrm{C}}},
$$

and this is six fifths of the cnergy when the charge is on the surface.

It therefore follows, by $\S 15$, that the values of $U$, T, and M for a sphere with a rolume-charge are six fifths as great as for the same sphere with an equal surface-charge*.

$\$ 21$. Pair of concentric spheres with uniformly distriluted complementary charges.-Let the outer sphere have the radius $a$ and the charge $Q$, while the inner sphere has the radius $b$ and the charge $-Q$. Then from $z=-a$ to $z=-b$ and also. from $z=b$ to $z=a$,

$$
d \mathrm{Q} / d z=\mathrm{Q} / 2 a,
$$

but from $z=-b$ to $z=b$

$$
\begin{gathered}
d \mathrm{Q} / d z=-\mathrm{Q}(a-b) / 2 a b . \\
\text { Thus } \int_{-a}^{+a}\left(\frac{d \mathrm{Q}}{d z}\right)^{2} d z=\frac{\mathrm{Q}^{2}(a-b)}{2 a^{2}}+\frac{\mathrm{Q}^{2}(a-b)^{2}}{2 a^{2} b}=\frac{\mathrm{Q}^{2}}{2} a \cdot \frac{a-b}{b} .
\end{gathered}
$$

For the outer sphere alone

$$
\int_{-a}^{+a}\left(\frac{d Q^{2}}{d z}\right)^{2} d z=\frac{Q^{2}}{2 a}
$$

and the value of $\mathrm{U}_{0}$ for the pair of spheres is $\mathrm{Q}^{2}(a-b) / 2 a b \mathrm{~K}$. Thus the values of $\mathrm{W}, \mathrm{P}$, and $\mathrm{U}_{0}$ for the system are $(a-b) / 6$

* Dr. M. Abraham has proved similar results for an ellipsoid by a method not depending on the calculation of $W$ and $P$ for the pulse. See his Theorie der Elektrizität, vol. ii. p. 170. 
times the values for the outer sphere alone, and hence, by $\$ 15$, the values of $\mathrm{U}, \mathrm{T}$, and $\mathrm{M}$ are $(a-b) / b$ times the values for the outer sphere alone.

In this calculation a uniform distribution of the charges has been assumed. But this is not the natural distribution, corresponding to the speed $u$, for a pair of conducting spheres. So far as I know, the distribution in the latter case has not been investigated.

\$22. Sphere with surface-density proportional to $\cos \theta$. Let $\sigma$, the surface-density, be given by $\sigma=\sigma_{0} \cos \theta$. Then the same distribution can be obtained by taking two spheres of volume-densities $\rho$ and $-\rho$ with their centres at a distance $\xi$ apart, and proceeding to the limit $\xi=0$, while $\rho \xi$ bas the constant value $\sigma_{0}$. In terms of $g$, the "electric moment" of the sphere, we have

$$
\rho \xi=\sigma_{0}=\frac{3 g}{4 \pi a^{3}} .
$$

Let $O$ (fig. 3 ) be the centre of the sphere of density $\rho$ and $O^{\prime}$ that of the sphere of density - $\rho$. Then, if $\mathrm{A}$ be the area of the section of the sphere $\mathrm{O}$ by a plane at a distance $z$ from $O$, the normal making an angle $\theta$ with $O X$, we have $\mathrm{A}=\pi\left(a^{2}-z^{2}\right)$. The area of the section of the sphere $O^{\prime}$ by the same plane is $\pi\left\{a^{2}-(z+\xi \cos \theta)^{2}\right\}$. Thus the charge $d Q$ contained between two planes at a distance $d z$ apart is

Fig. 3.

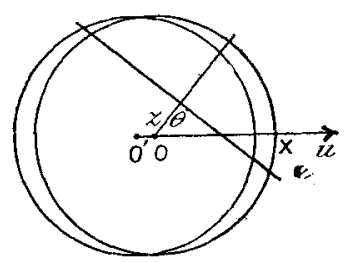

$$
d \mathrm{Q}=\pi \rho\left\{a^{2}-z^{2}\right\} d z-\pi \rho\left\{a^{2}-(z+\xi \cos \theta)^{2}\right\} d z,
$$

whence, to the first power of $\xi$, we have

$$
d \mathrm{Q} / d z=2 \pi z \cos \theta \cdot \rho \xi .
$$

Hence, since $\rho \xi=3 q / 4 \pi a^{3}$,

$$
\frac{d \mathrm{Q}}{d z}=\frac{3 \mu z \cos \theta}{2 \alpha^{3}} .
$$

Since

$$
\int_{-a}^{2+a} \hat{i}^{2} d z=\frac{2}{3} d^{3}
$$

we have, by (37),

$$
W=\frac{3 \mu u^{2} y^{2}}{4 u^{3}} \int_{0}^{\pi} \frac{\sin ^{3} \theta \cos ^{2} \theta d \theta}{(1-n \cos \theta)^{2}} .
$$


Putting $1-n \cos \theta=h$, we find

$$
\begin{aligned}
& W=\frac{3 \mu u^{2} g^{2}}{4 a^{3} n^{5}} \int_{1-\mu}^{1+n}\left\{\frac{n^{2}-1}{h^{2}}+\frac{4-\hat{z} n^{2}}{h}-\left(6-n^{2}\right)+4 h-h^{2}\right\} d h, \\
& =\frac{3 \mu u^{2} g^{2}}{2 a^{3}}\left\{\begin{array}{l}
v^{5} \\
u^{5}
\end{array}\left(2-\frac{u^{2}}{u^{2}}\right) \log \frac{v+u}{v-u}-4^{v^{4}}{ }_{u^{4}}+\frac{2}{3} v^{2} u^{2}\right\},
\end{aligned}
$$

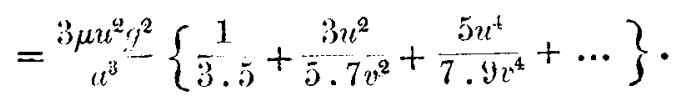

Since the sphere moves along an axis of symmetry, the momentum carried off by the pulse is given by (38), and thus, after integrating with respect to $z$, we have

$$
\begin{aligned}
P & =\frac{3 \mu u g^{2} n}{4 a^{3}} \int_{0}^{\pi} \frac{\sin ^{3} \theta \cos ^{3} \theta d \theta}{(1-n \cos \theta)^{2}} \\
& =\frac{3 \mu u g^{2}}{4 a^{3} n^{3}} \int_{-n}^{1+n}\left\{\frac{n^{2}-1}{h^{2}}+\frac{5-3 n^{2}}{h}-\left(10-3 n^{2}\right)+\left(10-n^{2}\right) h-5 h^{2}+h^{3}\right\} d l, \\
& =\frac{3 \mu u g^{2}}{4 a^{3} n^{2}}\left\{\left(5-3 n^{2}\right) \log _{1} \frac{1+n}{1-n}-10 n+3 n^{3}\right\} \\
& =\frac{6 \mu u^{3} g^{2}}{u^{3} v^{2}}\left\{\frac{1}{5.7}+\frac{2 u^{2}}{7.9 r^{2}}+\frac{3 u^{4}}{9.11 r^{4}} \cdots\right\} .
\end{aligned}
$$

When the sphere is at rest, the electrostatic potential at its surface is $\mathrm{V}=y \cos \theta / \mathrm{K} a^{2}$, and hence

$$
\begin{aligned}
\mathrm{U}_{0} & ={ }_{2} \Sigma V_{Y}=\frac{1}{2} \int_{0}^{\pi} \frac{g \cos \theta}{\mathrm{K} a^{2}} \cdot \sigma_{0} \cos \theta \cdot 2 \pi a^{2} \sin \theta d \theta \\
& =\frac{g^{2}}{2 a^{3} \mathrm{~K}}=\frac{\mu g^{2} v^{2}}{2 a^{3}} .
\end{aligned}
$$

From these values of $\mathrm{W}, \mathrm{P}$, and $\mathrm{U}_{0}$ we find, by $\S 1 \tilde{5}$, that for the sphere in steady motion,

$$
\begin{aligned}
& \mathrm{U}=\frac{\mu q^{2} v^{2}}{2 u^{3}}\left\{1+\frac{6 u^{4}}{5.7 v^{4}}+\frac{12 u^{6}}{7.9 v^{6}}+\frac{18 u^{8}}{9.11 v^{3}}+\ldots\right\} \\
& \mathrm{T}=\frac{3 \mu u^{2} u^{2}}{u^{3}}\left\{\frac{1}{3.5}+\frac{2 u^{2}}{5.7 v^{2}}+\frac{3 u^{1}}{7.9 v^{4}}+\frac{4 u^{6}}{9.11 v^{6}}+\ldots\right\} \\
& \mathrm{I}=\frac{6 \mu u q^{2}}{a^{3}} \cdot\left\{\frac{1}{3.5}+\frac{2 u^{2}}{5.7 v^{2}}+3.9 u^{4}+\frac{4 u^{6}}{9 \cdot 11 v^{6}}+\ldots\right\} .
\end{aligned}
$$


\$23. Ellipsoid of revolution with a surface-charge.-When a charged ellipsoid is in uniform motion, the charge is distributed in the same way as when it is at rest*. The surfacedensity is everywhere proportional to the thickness of an infinitely thin cllipsoidal shell bounded by similar ellipsoids, the inner surface of the shell coinciding with the ellipsoicl. Such a shell can he formed by uniformly straining a shell bounded by concentric spheres. If the spherical shell be cut by a series of parallel equidistant planes, they will divide the shell into portions of equal volume, and hènce the series of parallel equidistant planes, into which the first series is transformed by the strain, divides the ellipsoidal shell into portion: of equal volume. Thus, the charge contained between a pair of parallel planes which cut the ellipsoid is, for any given direction of the planes, proportional to the distance between them. Hence, if the distance between a pair of parallel tangent planes $\mathrm{AA}^{\prime}, \mathrm{BB}^{\prime}$ (fig. 4) be $p$, the value of $d \mathrm{Q} / d z$, where $d z$ is an element

Fig. 4.

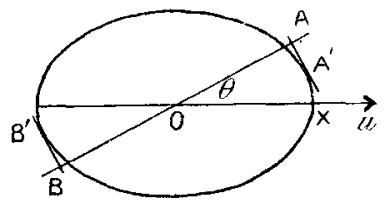
of the normal to these planes, is constant for those planes and is equal to $\mathrm{Q} / p$, where $\mathrm{Q}$ is the total charge on the ellipsoid.

$\$ 24$. For simplicity, the ellipsoid of revolution will be supposed to move along $\mathrm{OX}$, its axis of revolution. If $a, b$, $b$ be the axes of the ellipsoid, then, when the normal to the tangent planes makes an angle $\theta$ with $\mathrm{OX}$

$$
p^{2}=4\left\{b^{2}+\left(a^{2}-b^{2}\right) \cos ^{2} \theta\right\} .
$$

Since $d \mathrm{Q} / d z$ is independent of $z$, we can integrate (37) at once with respect to $z$, and thus, writing $\cos \theta=x$, we find for the energy carried off in the pulse

$$
\begin{aligned}
W & =\frac{\mu u^{2} \mathrm{Q}^{2}}{4} \int_{-1}^{+1} \frac{\left(1-u^{2}\right)}{(1-n x)^{2} \sqrt{l^{2}+\left(a^{2}-b^{2}\right) c^{2}}} \\
& =\frac{\mu u \imath^{2} \mathrm{Q}^{2}}{4 n^{2}}\left\{-\mathrm{J}_{1}+2 \mathrm{~J}_{2}-\left(1-n^{2}\right) \mathrm{J}_{3}\right\},
\end{aligned}
$$

where

$$
\begin{aligned}
& \mathrm{J}_{1}=\int_{-1}^{+1} \frac{d u}{\sqrt{ } b^{2}-i-\left(a^{2}-b^{2}\right) \cdot x^{2}}, \quad \mathrm{~J}_{2}=\int_{-1}^{+1} \frac{d x}{(1-n x) \sqrt{b^{2}+\left(a^{2}-b^{2}\right) x^{2}}} \\
& \text { and } \\
& J_{3}=\int_{-1}^{+1} \frac{d x^{2}}{(1-n x)^{2} \sqrt{ }^{2}+\left(a^{2}-b^{2}\right) \cdot c^{2}} .
\end{aligned}
$$

* W. B. Morton, Proc. Physical Society, vol. xiv. p. 180 (1896), or Searle, Phil. Mag. Oct. 1897, p. 331. 
Impulsive Motion of Electrified Systems.

When $a>b$, we put $a^{2}-b^{2}=c^{2}$, and then we have

$$
\mathbf{J}_{1}=\frac{1}{c} \log \frac{a+c}{a-c} .
$$

The case when $a<b$ is considered in $\S 26$.

If in $J_{2}$ we put $b^{2}\left(1-n^{2}\right)=a^{2}-l^{2}$, and change the variable from $x$ to $y$, where

$$
y+\frac{l^{2}-n^{2} a^{2}}{\left(1-n^{2}\right) l^{2}}=\frac{1}{1-n x},
$$

we find, after some reductions,

$$
\mathrm{J}_{2}=\frac{1}{\sqrt{l^{\overline{2}}}} \int \frac{d y}{\mathrm{R}}
$$

where

$$
\mathrm{R}^{2}=y^{2}+\frac{n^{2}\left(u^{2}-l^{2}\right)\left(l^{2}-n^{2} a^{2}\right)}{\left(1-n^{2}\right)^{2} 7^{4}} .
$$

Thus, when $l^{2}$ is positive, or $a^{2}>\left(1-n^{2}\right) b^{2}$, that is, when the ellipsoid is prolate or less oblate than the Heariside ellipsoid,

$$
J_{2}=\frac{1}{l}[\log (y+\mathrm{R})]_{x=-1}^{x=1}=\frac{1}{l} \log \frac{a+l}{t-l} .
$$

The case when $l^{2}$ is negative is considered in $\S 26$.

The third integral $J_{3}$ can now be easily found, for on differentiating the integral $J_{2}$ with regard to $n$, we find that $n d \mathrm{~J}_{2} / d n=\mathrm{J}_{3}-\mathrm{J}_{3}$. Thus

$$
\mathrm{J}_{3}=\frac{d}{d n}\left(n \mathrm{~J}_{2}\right)
$$

Since $d l / d n=n b^{2} / l$, we find

$$
\mathrm{J}_{3}=\frac{l^{2}-n^{2} l^{2}}{l^{3}} \log \frac{a+l}{a-l}+\frac{2 a n^{2} l^{2}}{l^{2}\left(\bar{a}^{2}-l^{2}\right)} .
$$

Collecting these resutts, and using $\mu \mathrm{K} v^{2}=1$, we have

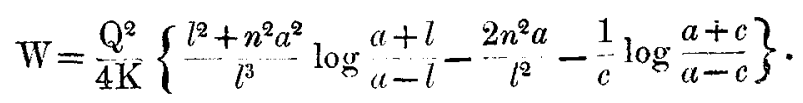

The electrostatic cnergy of the ellipsoid at rest is $\mathrm{U}_{0}$, where

$$
\mathrm{U}_{0}=\frac{\mathrm{Q}^{2}}{4 \mathrm{~K} c} \log \frac{a+c}{a-c} .
$$

$\S 25$. With the notation of $\S 24$, the momentum carried 
off by the pulse is given by

$$
\begin{aligned}
\mathrm{P} & =\frac{\mu u n \mathrm{Q}^{2}}{4} \int_{-1}^{+1} \frac{\left(1-x^{2}\right) x d x}{(1-n x)^{2} \sqrt{l^{2}+\left(a^{2}-l^{2}\right) x^{2}}} \\
& =\frac{\mu u \mathrm{Q}^{2}}{4 n^{2}} \cdot\left\{-2 \mathrm{~J}_{1}+\left(3-n^{2}\right) \mathrm{J}_{2}-\left(1-n^{2}\right) \mathrm{J}_{3}-n \mathrm{~J}_{4}\right\},
\end{aligned}
$$

where

$$
J_{1}=\int_{-.1}^{+1} \frac{x d x}{\sqrt{b^{2}+\left(u^{2}-b^{2}\right) x^{2}}}=0
$$

Thus, using $\mu \mathrm{K} r^{2}=1$, we find

$$
\mathrm{P}=\frac{\mathrm{Q}^{2}}{4 \mathrm{~K} u}\left\{\frac{n^{2} l^{2}+2 l^{2}-n^{2} l^{2}}{l^{3}} \log \frac{a+l}{a-l}-\frac{2 a n^{2}}{l^{2}}-\frac{2}{c} \log \frac{a+c}{a-c}\right\} .
$$

By $\S 15$ we obtain the results

$$
\begin{aligned}
& \mathrm{U}=\frac{\mathrm{Q}^{2}}{4 \mathrm{~K}}\left\{\frac{n^{2} a^{2}+2 l^{2}-n^{2} l^{2}}{2 l^{3}} \log \frac{a+l}{a-l}-\frac{a n^{2}}{l^{2}}\right\}, \\
& \mathrm{T}=\frac{u^{2} \mathrm{Q}^{2}}{4 \mathrm{~K}{t^{2}}^{2}}\left\{\frac{a^{2}+l^{2}}{2 l^{3}} \log \frac{a+l}{a-l}-\frac{a}{l^{2}}\right\} .
\end{aligned}
$$

By (27), M=2T/ 1 .

These values of $\mathrm{U}$ and $\mathrm{T}$ agree with those $\mathrm{I}$ obtained by integration throughout the whole field of the moving ellipsoid *.

$\S 26$. When $a<b$, the quantity $c$ is imaginary, and when $a$ becomes still smaller, so that $a<b \sqrt{1-n^{2}}$, the quantity $l$ is imaginary also. The last case corresponds to an ellipsoid more oblate than Heaviside's. The expressions for $\mathrm{U}_{0}, \mathrm{~W}$, and $P$ can be adapted to the case where $b\left(1-n^{2}\right)^{\frac{1}{2}}<a<b$, and where $c$ is imaginary but $l$ is real, by putting $b^{2}-a^{2}=\mathrm{C}^{2}$ and writing

$$
\frac{2}{\mathrm{C}} \tan ^{-1} \frac{\mathrm{C}}{a} \text { for } \frac{1}{c} \log \frac{a+c}{a-c} \text {. }
$$

When $a<b\left(1-n^{2}\right)^{\frac{2}{2}}$, both $c$ and $l$ are imaginary and we must, in addition, put $b^{2}\left(1-n^{2}\right)=\pi^{2}=\mathrm{L}^{2}$ and write

$$
\frac{2}{\mathrm{~L}} \tan ^{-1} \frac{\mathrm{L}}{a} \text { for } \frac{1}{l} \log \frac{a+l}{a-l} \text {. }
$$

For a disk of radius $b$, we put $a=0$ and hence $C=b$ and $\mathrm{L}=h,\left(1-n^{2}\right)^{\frac{1}{2}}$. We thus obtain

$$
\begin{gathered}
\mathrm{U}_{0}=\frac{\pi \mathrm{Q}^{2}}{4 \mathrm{~K} b}, \quad \mathrm{~W}=\frac{\pi \mathrm{Q}^{2}}{4 \mathrm{~K} b}\left(\frac{1}{\left(1-n^{2}\right)^{\frac{2}{2}}}-1\right), \\
\mathrm{P}=\frac{\pi \mathrm{Q}^{2}}{4 \mathrm{~K} b u}\left(\frac{2-n^{2}}{\left(1-n^{2}\right)^{\frac{2}{2}}}-2\right) . \\
* \text { Plil. Mag. Oct. 1897, p. } 340 .
\end{gathered}
$$


By the results of $\S 15$ we obtain

$$
\begin{aligned}
& \mathrm{U}=\frac{1}{2} u \mathrm{P}+\mathrm{U}_{0}=\mathrm{U}_{0} \frac{1-\frac{1}{2} n^{2}}{\left(1-n^{2}\right)^{\frac{1}{2}}}=\mathrm{U}_{0}\left(1+\frac{1}{8} n^{4}+\ldots\right), \\
& \mathrm{T}=\mathrm{W}-\frac{1}{2} u \mathrm{P}=\mathrm{U}_{0} \frac{n^{2}}{2\left(1-n^{2}\right)^{\frac{1}{2}}}=n^{2} \mathrm{U}_{0}\left(\frac{1}{2}+\frac{1}{4} n^{2}+\ldots\right) .
\end{aligned}
$$

If we put $n=\sin \gamma$, we obtain

$$
\begin{gathered}
\mathrm{U}=\frac{1}{2}(\sec \gamma+\cos \gamma) \mathrm{U}_{0}, \\
n_{t}=2 \mathrm{~T} / u^{2}=\sec \gamma \cdot \mathrm{U}_{0} / v^{2}, \quad m_{l}=2 d(\mathrm{~T} / u)^{\prime} / d u=\sec ^{3} \gamma \cdot \mathrm{U}_{0} / v^{2},
\end{gathered}
$$

where $m_{t}$ is the "transverse mass" and $m_{l}$ the "longitudinal mass."

\$27. Circular disk moving in its own plane.-In this instance the axis of symmetry of the system is not parallel to the direction of motion, as is the case in the other problems we have considered. We therefore require two angular coordinates, $\phi$ and $\psi$, where $\phi$ is measured from the axis of the disk and $\psi$ is measured round the axis, starting from the plane which contains the axis, and is parallel to the direction of motion. Then, if the radius defined by $\phi$ and $\psi$ makes the angle $\theta$ with the direction of motion, we have

$$
\cos \theta=\sin \phi \cos \psi
$$

Taking the disk, of radius $b$, as the limiting form of an ellipsoid, we see that $p$, the thickness of the pulse, is equal to $2 b \sin \phi$, and thus, by $\$ 23, d \mathrm{Q} / d z=\mathrm{Q} / 2 b \sin \phi$. Now $d \omega$; the element of solid angle, is equal to $\sin \phi d \phi d \psi$ and thus, by $(34)$, since $d Q / d z$ is independent of $z$,

$$
\begin{aligned}
W & =\frac{\mu \iota^{2} Q^{2}}{8 \pi b} \iint \frac{\sin ^{2} \theta d \phi d \psi}{(1-n \cos \theta)^{2}} \\
& =\frac{\mu \iota^{2} Q^{2}}{\delta \pi b} \iint \frac{\left(1-\sin ^{2} \phi \cos ^{2} \psi\right)}{(1-n \sin \phi \cos \psi)^{2}} d \phi d \psi,
\end{aligned}
$$

where $\phi$ goes from 0 to $\pi$ and $\psi$ from 0 to $2 \pi$.

Expanding the denominator and integrating term by term, we find

$$
\mathrm{W}=\frac{\pi \mu u^{2} \mathrm{Q}^{2}}{4 b}\left\{\frac{3}{1}\left(\frac{1}{2}\right)^{2}+n^{2} \cdot \frac{7}{3}\left(\frac{1.3}{2.4}\right)^{2}+n^{4} \cdot \frac{11}{5}\left(\frac{1.3 .5}{2 \cdot 4 \cdot 6}\right)^{2}+\ldots\right\} .
$$

We can express this result in a finite form by means of the first and second complete elliptic integrals $F$ and $E$. If $\Delta^{2}=1-n^{2} \sin ^{2} \alpha$, we have

$$
\begin{aligned}
& \mathrm{F}=\int_{0}^{\frac{\pi}{2}} \frac{d \alpha}{\Delta}=\frac{\pi}{2}\left\{1+n^{2}\left(\frac{1}{2}\right)^{2}+n^{4}\left(\frac{1.3}{2.4}\right)^{2}+n^{6}\left(\frac{1 \cdot 3 \cdot 5}{2 \cdot 4 \cdot 6}\right)^{2}+\ldots\right\} \\
& \mathrm{E}=\int_{0}^{\frac{\pi}{2}} \Delta d \alpha=\frac{\pi}{2}\left\{1-\frac{n^{2}}{1}\left(\frac{1}{2}\right)^{2}-\frac{n^{4}}{3}\left(\frac{1.3}{2.4}\right)^{2}-\frac{n^{6}}{5}\left(\frac{1.3 .5}{2 \cdot 4 \cdot 6}\right)^{2}+\ldots\right\} .
\end{aligned}
$$


Comparing the series for $W$ with those for $\mathrm{F}$ and $\mathrm{E}$ and using $\mu \mathrm{K} v^{2}=1$, we obtain

$$
\mathrm{W}=\frac{\mathrm{Q}^{3}}{2 b \mathrm{~K}}\left(2 \mathrm{~F}-\mathrm{E}-\frac{1}{2} \pi\right) .
$$

The momentum carried off by the pulse is, by symmetry, parallel to the direction of motion and hence, by (36), is given by

$$
\begin{aligned}
\mathrm{P} & =\frac{\mu u n \mathrm{Q}^{2}}{8 \pi b} \iint \frac{\sin ^{2} \theta \cos \theta d \phi d \psi}{(1-u \cos \theta)^{2}} \\
& ={ }^{\mu u n \mathrm{Q}^{2}} \iint_{8 \pi} \int_{\xi} \frac{\sin \phi \cos \psi-\sin ^{3} \phi \cos ^{3} \psi}{(1-u \sin \phi \cos \psi)^{2}} d \phi d \psi,
\end{aligned}
$$

where, as before, $\phi$ goes from 0 to $\pi$ and $\psi$ from 0 to $2 \pi$. Expanding the denominator and integrating term by term, we find

$$
\mathrm{P}=\frac{\pi \mu u n^{2} \mathrm{Q}^{2}}{4 \bar{b}}\left\{2 \cdot \frac{7}{4^{2}}\left(\begin{array}{l}
1 \\
2
\end{array}\right)^{2}+4 n^{2} \frac{11}{6^{2}}\left(\frac{1.3}{2.4}\right)^{2}+6 n^{4} \frac{15}{8^{2}}\left(\frac{1.3 .5}{2.4 .6}\right)^{2}+\ldots\right\} .
$$

Thus, as was proved in $\S 18$, the momentum carried off in the pulse is proportional to $u^{3}$ for small values of $u / v$. Comparing the series for $\mathrm{P}$ with those for $\mathrm{F}$ and $\mathrm{E}$ and using $\mu \mathrm{K} v^{2}=1$, we obtain

$$
\mathrm{P}=\frac{\mathrm{Q}^{2}}{2 l, \overline{\mathrm{K}} u}\left\{\left(3-n^{2}\right) \mathrm{F}-\mathrm{E}-\pi\right\} .
$$

Since the momentum of the field, when the disk is in steady motion, is parallel to the direction of $u$, we can employ the formula of $\S 15$. Thus, since

we obtain

$$
\mathrm{U}_{0}=\pi \mathrm{Q}^{2} / 4 b \mathrm{~K},
$$

$$
\begin{aligned}
& \mathrm{U}=\frac{1}{2} u \mathrm{P}+\mathrm{U}_{0}=\mathrm{U}_{0} \pi^{-1}\left\{\left(3-n^{2}\right) \mathrm{F}-\mathrm{E}\right\}=\mathrm{U}_{0}\left(1+7 n^{4} / 64+\ldots\right), \\
& \mathrm{T}=\mathrm{W}-\frac{1}{2} u \mathrm{P}=\mathrm{U}_{0} \pi^{-1}\left\{\left(1+n^{2}\right) \mathrm{F}-\mathrm{E}\right\}=n^{2} \mathrm{U}_{0}\left(3 / 4+7 n^{2} / 32+\ldots\right) .
\end{aligned}
$$

Comparing these results with those of $\S 26$, we see that the magnetic energy of a disk moving in its own plane is, for small speeds, greater than the magnetic energy of the disk moving parallel to its axis in the proportion of 3 to 2 .

$\S 28$. To show how the increase of speed affects the electric and magnetic energies when the disk moves (1) along its axis, (2) in its plane, the following table is given. Since the "transverse mass," $m_{t}$, is equal to $2 \mathrm{~T} / u^{2}$, it will be convenient to tabulate $m / / m_{0}$, where- $m_{0}$ is the value of $m_{t}$ for infinitesimal 
speeds. To facilitate the use of Legendre's tables* for $\mathbf{E}$ and $\mathrm{F}$, we pat $n=\sin \gamma$.

\begin{tabular}{|c|c|c|c|c|c|}
\hline \multirow[b]{2}{*}{$\gamma$} & \multirow[b]{2}{*}{$n$. } & \multicolumn{2}{|c|}{ Motion along Axis. } & \multicolumn{2}{|c|}{ Motion perpendicular to Axis. } \\
\hline & & $\mathbf{U} / \mathbf{U}_{0}$ & $m_{t} / m_{0}$ & $\mathbf{U} / \mathrm{U}_{0}$ & $m_{t} / m_{0}$ \\
\hline 10 & $\cdot 1736$ & 1.0001 & 10154 & $1 \cdot 0001$ & $1 \cdot 0106$ \\
\hline 20 & $\cdot 3420$ & 1.0019 & 1.0642 & 1.0016 & 1.0366 \\
\hline 30 & .5000 & $1 \cdot 0104$ & $1 \cdot 1547$ & 1.0085 & 1.0860 \\
\hline 40 & $\cdot 6428$ & 1.0357 & $1 \cdot 3054$ & 10278 & $1 \cdot 1627$ \\
\hline 50 & 7660 & $1 \cdot 0992$ & 1.5557 & 10712 & 1.2772 \\
\hline 60 & .8660 & 1.2500 & 20000 & $l \cdot 1590$ & 14502 \\
\hline 70 & .0397 & $1 \cdot 6329$ & $2 \cdot 9238$ & $1 \cdot 3317$ & $1 \cdot 7291$ \\
\hline 80 & .0848 & 29662 & $5 \cdot 7588$ & 17068 & 22631 \\
\hline 85 & .9962 & 5.7804 & $11 \cdot 4737$ & $2 \cdot 1263$ & 28830 \\
\hline
\end{tabular}

We notice that, as $n$ increases, both $\mathrm{U} / \mathrm{U}_{0}$ and $m_{t} / m_{0}$ increase more rapidly when the motion is along the axis of. the disk than when it is perpendicular to the axis. For some value of $n$ between 8660 and 9397 the value of $m_{t} / m_{0}$ for motion along the axis becomes three halves of the value of $m_{t} / m_{0}$ for motion perpendicular to the axis, and then the magnetic energy for motion along the axis becomes equal to that for motion perpendicular to the axis.

The "longitudinal mass," $m_{l}$, is easily found. For we have $\dagger$

and thus

$$
\frac{d \mathrm{~F}}{d n}=\frac{\mathrm{E}}{n\left(1-n^{2}\right)}-\frac{\mathrm{F}}{n}, \quad \frac{d \mathrm{E}}{d n}=\frac{\mathrm{E}-\mathrm{F}}{n}
$$

$$
n_{l}=2 \frac{d}{d u}\left(\frac{\mathrm{T}}{u}\right)=\frac{2}{v^{2}} \frac{d}{d n}\left(\frac{\mathrm{T}}{n}\right)=\frac{2 \mathrm{U}_{0}}{v^{2}} \cdot \frac{\left(1+n^{2}\right) \mathrm{E}-\left(1-n^{2}\right) \mathrm{F}}{\pi n^{2}\left(1-n^{2}\right)} .
$$

\$29. Momentum and energy of a system of revolution moving slowly in any divection.-When a system which is symmetrical round an axis, and is also symmetrical with respect to a plane normal to that axis, moves either along or at right angles to its axis, its electromagnetic momentum is in the direction of motion, but the amounts of momentum are different in the two cases. If, however, the axis makes any angle other than 0 or $\frac{1}{2} \pi$ with the direction of motion, the momentum has a component at right angles to that direction as well as one parallel to it.

A general investigation would be difficult, but we can easily

* Legendre, Traitê des Fonctions Elliptiques, vol. ii.

+ A. Caley, "Elementary Treatise on Elliptic Functions," chapter 3. 
obtain some interestingr results when $n$ or $u / v$ is so small that, as we see by (1), the electric force due to the system may be regarded as being the same as if the system were at rest. The axis of $x$ will be taken in the direction of motion, the volume integral of $\mathrm{E}_{1}{ }^{2}$ through all space will be denoted $b$. $\Sigma \mathrm{E}_{1}^{2}$, and $\eta$ will be written for $\mathrm{K} / 4 \pi v^{2}$, or $\mu \mathrm{K}^{2} / 4 \pi$.

If $\mathrm{M}_{1}, \mathrm{M}_{2}, \mathrm{M}_{3}$ be the components of the momentum when the axis of the system is parallel to the axis of $x$, we have, by (24), since $\mu \mathrm{K} v^{2}=1$

$\mathrm{M}_{1}=u \eta \Sigma\left(\mathrm{E}_{2}{ }^{2}+\mathrm{E}_{3}{ }^{2}\right)=u \eta \Sigma\left(\mathrm{E}^{2}-\mathrm{E}_{1}{ }^{2}\right)=2\left(u_{i}^{\prime} v^{2}\right) \mathrm{U}_{0}-u \eta \Sigma \mathrm{E}_{1}{ }^{2}(40)$ while, by (26), we have, for reasons of symmetry,

$$
\mathrm{M}_{2}=-u \eta \Sigma \mathrm{E}_{1} \mathrm{E}_{2}=0, \quad \mathrm{M}_{3}=-u \eta \Sigma \mathrm{E}_{1} \mathrm{E}_{3}=0 .
$$

In the expression for $M_{1}$ we have put $U_{0}$ for $U$, since we know, by $\S 18$, that, when $n$ is small, $\mathrm{U}-\mathrm{U}_{0}$ is proportional to $n^{4}$. Since there is symmetry round the axis, $\Sigma \mathrm{E}_{2}^{2}=\Sigma \mathrm{E}_{3}{ }^{2}$, and thus

$$
u \eta \Sigma \mathrm{E}_{1}^{2}=2 u \mathrm{U}_{0} / v^{2}-\mathrm{M}_{1}, \quad u \eta \Sigma \mathrm{E}_{2}{ }^{2}=u \eta \Sigma \mathrm{E}_{3}{ }^{2}=\frac{1}{2} \mathrm{M}_{1} .
$$

Now let the system be turned round the axis of $y$ in the positive direction, so that the axis of the system makes an angle $\theta$ with the direction of motion and with the axis of $x$, and let $G$ be a point rigidly connected with the system and turning with it. Since when $u / v$ is very small the electric field turus round with the system without any other change, the new components of the electric force at $G$ are given by the equations

$$
\left.\begin{array}{l}
\mathrm{E}_{1}^{\prime}=\mathrm{E}_{1} \cos \theta+\mathrm{E}_{3} \sin \theta, \quad \mathrm{E}_{2}{ }^{\prime}=\mathrm{E}_{2} \\
\mathrm{E}_{3}^{\prime}=-\mathrm{E}_{1} \sin \theta+\mathrm{E}_{3} \cos \theta
\end{array}\right\} .
$$

For $\mathrm{M}_{1}{ }^{\prime}$, the component of the momentum in the direction of motion, we have, as in (40),

$$
\mathrm{M}_{1}{ }^{\prime}=2 u \mathrm{U}_{0} / v^{2}-u \eta \Sigma \mathrm{E}_{1}{ }^{\prime 2}
$$

But $\Sigma \mathrm{E}_{1} \mathrm{E}_{3}=0$, and hence, by (43),

$$
\begin{aligned}
\mathrm{M}_{1}^{\prime} & =2 u \mathrm{U}_{0} / v^{2}-u \eta \Sigma\left(\mathrm{E}_{1}^{2} \cos ^{2} \theta+\mathrm{E}_{3}^{2} \sin ^{2} \theta\right), \\
& =2 u \mathrm{U}_{0} \sin ^{2} \theta / v^{2}+\mathrm{M}_{1}\left(\cos ^{2} \theta-\frac{1}{2} \sin ^{2} \theta\right) .
\end{aligned}
$$

If $\mathrm{N}$ be the momentum in the divection of motion when the system moves at right angles to its axis, $i$. e., when $\theta=12 \pi$, we have

$$
\mathrm{N}=2_{2 u} \mathrm{U}_{0} / r^{2}-\frac{1}{2} \mathrm{M}_{1}, \quad \text {. . . . . }
$$

and thus for any value of $\theta$,

$$
\mathrm{M}_{1}^{\prime}=\mathrm{M}_{1} \cos ^{2} \theta+\mathrm{N} \sin ^{2} \theta .
$$


By symmetry $\Sigma \mathrm{E}_{1} \mathrm{E}_{2}=0$, and $\Sigma \mathrm{E}_{2} \mathrm{E}_{3}=0$, and hence, by (43),

$$
\mathrm{M}_{2}{ }^{\prime}=-u \eta \Sigma \mathrm{E}_{1}{ }^{\prime} \mathrm{E}_{2}{ }^{\prime}=-u \eta \Sigma\left(\mathrm{E}_{1} \cos \theta+\mathrm{E}_{3} \sin \theta\right) \mathrm{E}_{2}=0 \text {. }
$$

The momentum at right angles to the direction of motion is thus given by

$$
\begin{aligned}
\mathrm{M}_{3}{ }^{\prime} & =-u \eta \Sigma \mathrm{E}_{1}{ }^{\prime} \mathbf{E}_{3}{ }^{\prime}=\imath \eta \mathrm{\Sigma}\left(\mathrm{E}_{1}{ }^{2}-\mathrm{E}_{3}{ }^{2}\right) \sin \theta \cos \theta \\
& =\left(\mathrm{N}-\mathrm{M}_{1}\right) \sin \theta \cos \theta . \quad . \quad . \quad . \quad . \quad .
\end{aligned}
$$

When $\theta=\frac{1}{2} \pi$, the component of the momentum at right angles to the direction of motion vanishes, and hence $N$ is the resultant momentum in that case.

From (45) we see that the resultant momentum is inclined to the direction of motion at an angle $\psi$, where

$$
\tan \psi=M_{3}{ }^{\prime} / M_{1}{ }^{\prime}=\left(N-M_{1}\right) \sin \theta \cos \theta /\left(M_{1} \cos ^{2} \theta+N \sin ^{2} \theta\right) \text {. }
$$

If $\mathrm{T}^{\prime}$ be the magnetic energy for any value of $\theta$, and ' $\mathrm{T}$ and $\mathrm{S}$ be the magnetic energies when the system moves along and at right angles to its axis, we have, by (25),

Thus

$$
\mathrm{T}^{\prime \prime}=\frac{1}{2} \mathrm{M}_{1}^{\prime} u, \quad \mathrm{~T}=\frac{1}{2} \mathrm{M}_{1} \pi, \quad \mathrm{S}=\frac{1}{2} \mathrm{~N}^{\prime} u .
$$

and

$$
\mathrm{T}^{\prime}=\mathrm{T} \cos ^{2} \theta+\mathrm{S} \sin ^{2} \theta, \quad \text {. . . . . }
$$

$$
\mathrm{S}=u^{2} \mathrm{U}_{0} / v^{2}-\frac{1}{2} \mathrm{~T} . \quad . \quad . \quad . \quad . \quad . \quad .
$$

$\S 30$. We may illustrate the formulæo of $\S 29$ by the results for a charged disk of radius $l$. Here we have, by $\$ 26$, for very small speeds

Hence

$$
\mathrm{U}_{0}=\pi \mathrm{Q}^{2} / 4 \mathrm{~K} l, \quad \mathrm{~T}=\frac{1}{2} \iota^{2} \mathrm{U}_{0} / r^{2} .
$$

$$
\mathrm{S}=u^{2} \mathrm{U}_{0} / v^{2}-\frac{1}{2} \mathrm{~T}^{\prime}=\frac{3}{4} u^{2} \mathrm{C}_{\mathrm{o}} / v^{2},
$$

and thus the magnetic energy of the disk moving slowly in its own plane is half as great again as when it moves along its axis at the same speed, as we found in $\$ 27$.

Now $\mathrm{N}-\mathrm{M}_{1}=2(\mathrm{~S}-\mathrm{T}) / u=\frac{1}{2} u \mathrm{U}_{0} / v^{2}$, and hence, when the axis of the disk makes an angle $\theta$ with the direction of motion,

$$
\begin{aligned}
& \mathrm{M}_{1}^{\prime}=\mathrm{M}_{1}+\left(\mathrm{N}-\mathrm{M}_{1}\right) \sin ^{2} \theta=\frac{1}{2}\left(u / v^{2}\right) \mathrm{U}_{0}\left(2+\sin ^{2} \theta\right), \\
& \mathrm{M}_{3}{ }^{\prime}=\frac{1}{2}\left(u / v^{2}\right) \mathrm{U}_{0} \sin \theta \cos \theta .
\end{aligned}
$$

Thus the resultant momentum is inclined to the direction of motion at an angle $\psi$, where

$$
\operatorname{tin} \psi=\frac{\sin \theta \cos \theta}{2+\sin ^{2} \theta} \text {. }
$$


It is easily found that tan $\psi$ has a maximum value $(24)^{-\frac{1}{2}}$ or $0 \cdot 20412$, corresponding to $\psi=11^{\circ} 32^{\prime}$, when $\sin ^{2} \theta=2 / 5$ or $\theta=39^{\circ} 14^{\prime}$.

For a sphere with a surface-charge where the surfacedensity is $\sigma_{0} \cos \theta$, we have, by $\$ 22$, when $u / v$ is very small,

$$
\mathrm{T}=2 u^{2} \mathrm{U}_{0} / \check{\partial} v^{2} \text {. }
$$

Hence, by (47),

and

$$
\begin{aligned}
& \mathrm{S}=u^{2} \mathrm{U}_{0} / v^{2}-\frac{1}{2} \mathrm{~T}=4 u^{2} \mathrm{U}_{0} / \tilde{5} v^{2}=2 \mathrm{~T} \\
& \mathrm{~N}=2 \mathrm{M}_{1} .
\end{aligned}
$$

For a prolate ellipsoid, when $n$ is very small, we may put $l=c$, and then we find, by $\S 25$, that

Hence

$$
\mathrm{T}=\frac{u^{2} \mathrm{Q}^{2}}{4 \mathrm{~K} v^{2}}\left\{\frac{a^{2}+c^{2}}{2 c^{3}} \log \frac{a+c}{a-c}-\frac{a}{c^{2}}\right\} .
$$

$$
\begin{aligned}
\mathrm{S} & =u^{2} \mathrm{U}_{0} / v^{2}-\frac{1}{2} \mathrm{~T} \\
& =u^{2} \mathrm{Q}^{2} \\
4 \mathrm{~K} v^{2} & \left\{\frac{3 c^{2}-a^{2}}{4 c^{3}} \log \frac{a+c}{a-c}+\frac{a}{2 c^{2}}\right\}
\end{aligned}
$$

where $c^{2}=a^{2}-b^{2}$.

For a very slender ellipsoid, where $a / b$ is very great, we may put $(a+c) /(a-c)=4 a^{2} / b^{2}$, and need retain only the logarithmic terms. We then find

$$
\mathrm{T}=u^{2} \mathrm{U}_{0} / v^{2}, \quad \mathrm{~S}=\frac{1}{2} \mathrm{~T} \text {. }
$$

$\S 31$. Hitherto we have supposed that the speed is impulsively changed from 0 to $u$ or from $u$ to 0 . But the principle of $\S 3$ is easily extended to the case when the velocity of a point charge is impulsively changed from $\mathbf{u}$ to $\mathbf{u}^{\prime}$, where $\mathbf{u}$ and $\mathbf{u}^{\prime}$ may be inclined at any angle. We have, as Dr. Heaviside ${ }^{*}$ has indicated, only to destroy the velocity $u$ and then instantly restart the charge with the velocity $\mathfrak{u}^{\prime}$. By (8) we obtain a vector expression for $\mathbf{e}$ in the form

$$
\mathbf{e}=\frac{q}{\mathrm{~K} r}\left[\frac{\mathbf{r}_{1}\left(\mathbf{u}^{\prime} \mathbf{r}_{1}\right)-\mathbf{u}^{\prime}}{v-\mathbf{u}^{\prime} \mathbf{r}_{1}}-\frac{\mathbf{r}_{1}\left(\mathbf{u r}_{1}\right)-\mathbf{u}}{v-\mathbf{u r}_{1}}\right] . .
$$

Writing $\mathbf{a}^{\prime}-\mathfrak{u}=\mathbf{w}$ and putting $v-u \cos \theta=v-\mathrm{ur}_{1}=v$ we easily find

$$
e=\frac{q}{\mathrm{~K} r} \cdot\left[-\frac{\mathrm{w}}{v h-w \cos \gamma}+\frac{\left(v \mathbf{r}_{1}-\mathfrak{u}\right) w \cos \gamma}{v h(v / t-w \cos \gamma}\right] .
$$

Here $\theta$ is the angle between $r$ and $u$ while $y$ is the angle between $r$ and $w$.

* 'Nature,' Nov. 6, 1902. 
If $w$ be infinitesimal compared with $r-u,(49)$ becomes

$$
\mathbf{e}=\frac{\sigma}{\overline{\mathbf{K}} r}\left[-\frac{\mathrm{w}}{v h}+\frac{\left(r \mathbf{r}_{1}-\mathbf{u}\right) w \cos \gamma}{v^{2} h^{2}}\right] . \quad .
$$

Applying (50) to a sphere of radius $a$ with it uniform surface-charge $Q$ in the manner explained in $\$ 7$, we find that in the pulse, at a great distance from the sphere,

$$
\mathrm{E}=\frac{\mathrm{Q}}{2 \mathrm{~K} r(\ell}\left[-\frac{\mathrm{w}}{v / h}+\frac{\left(\imath \mathrm{r}_{1}-\mathfrak{u}\right) u \cos \gamma}{v^{2} l^{2}}\right] . .
$$

If $\psi$ be the angle between $\mathbf{u}$ and $\mathbf{w}$, we find for the energy per unit volume in the pulse, since $\mu \mathrm{K} r^{2}=1$ and $n=u / r$,

$\frac{\mathrm{KE}^{2}}{4 \pi}=\frac{\mu \mathrm{Q}^{2} w^{2}}{16 \pi r^{2} \iota^{2}}\left[\frac{1}{h^{2}}+\frac{2 n \cos \gamma \cos \psi}{h^{3}}-\frac{\left(1-n^{2}\right) \cos { }^{2} \gamma}{h^{4}}\right]$.

One angular coordinate of $r$ is $\theta$; if the other be $\phi$ and be measured round the direction of $u$ from the plane of $u$ and w, we have

$$
\cos \gamma=\cos \theta \cos \psi+\sin \theta \sin \psi \cos \phi .
$$

Fig. 5. The various angles are shown in fig. 5 .

$$
\begin{aligned}
& \text { The thickness of the pulse is } 2 a \text {, and } \\
& \text { hence the energy radiated away in the } \\
& \int_{0}^{2 \pi} \cos ^{2} \gamma d \phi=\frac{2 \pi}{2 n^{2}}\left\{\left(2-3 \sin ^{2} \psi\right)(1-h)^{2}+n^{2} \sin ^{2} \psi\right\} .
\end{aligned}
$$

Integrating with respect to $\phi$ from 0 to $2 \pi$, and writing $d h / n$ for $\sin \theta d \theta$, we find

$$
\begin{aligned}
& \mathrm{W}=\mathrm{A}-\mathrm{B} \sin ^{2} \Psi, \\
& \mathrm{A}=\frac{\mu \mathrm{Q} w^{2}}{4 a h^{3}} \int_{1-n}^{1+n}\left(-\frac{1}{h^{2}}+\frac{2}{h^{3}}-\frac{1-n^{2}}{h^{4}}\right) d h, \\
& \mathrm{~B}=\frac{\mu \mathrm{Q}^{2} w^{2}}{4 a n^{3}} \int_{1-n}^{1+n}\left\{-\frac{3+n^{2}}{2 h^{2}}+\frac{3-n^{2}}{l^{3}}-\frac{\left(1-n^{2}\right)\left(3-n^{2}\right)}{2 / l^{4}}\right\} d h .
\end{aligned}
$$

Phil. Mar. S. 6. Vol. 13. No. 73. Jan. 1907.

$\mathrm{L}$ 
Integrating with respect to $h$, we find

$$
W=\frac{\mu Q^{2} w^{2}}{3 a\left(1-u^{2}\right)^{2}}\left(1-u^{2} \sin ^{2} \psi\right)^{*} .
$$

$\S 32$. In the pulse, $\mathrm{H}=v \mathrm{KVr_{1 }} \mathbf{E}$, and thus the momentum per unit volume is $(\mathrm{K} / 4 \pi v) V \mathbf{E V \mathbf { r } _ { 1 }} \mathbf{E}$ or $\mathbf{r}_{1} \mathrm{KE}^{2} / 4 \pi v$, since $\mathbf{E}$ is perpendicular to $\mathrm{r}$. Hence, if $\mathrm{P}_{1}$ be the momentum in the pulse in the direction of $\mathbf{u}$,

$$
\mathrm{P}_{1}=\iint \frac{K E^{2}}{4 \pi v} 2\left(c r^{2} \cos \theta \sin \theta d \theta d \phi\right.
$$

Using the values of $\int \cos \gamma d \phi$ and $\int \cos ^{2} \gamma d \phi$ given in $\S 31$ and writing $(1-h) / n$ for $\cos \theta$, we find

$$
\begin{aligned}
& \mathrm{P}_{1}=\mathrm{C}-\mathrm{D} \sin ^{2} \psi \\
& \mathrm{C}=\frac{\mu \mathrm{Q}^{2} w^{2}}{4 a c n^{1}} \int_{1-n}^{0}\left\{\frac{1}{h}-\frac{3}{h^{2}}+\frac{3-n^{2}}{h^{3}}-1-n^{2} h^{+}\right\} d h \\
& \mathrm{D}=\frac{\mu \mathrm{Q}^{2} w^{2}}{4 a v n^{4}} \int_{1-n}^{1+n}\left\{\frac{3+n^{2}}{2 h}-\frac{9-n^{2}}{2 h^{2}}+\frac{\left(3-n^{2}\right)^{2}}{2 h^{3}}-\frac{\left(1-n^{2}\right)\left(3-n^{2}\right)}{2 h^{4}}\right\} d h .
\end{aligned}
$$

Integrating with respect to $h$, we find

$$
\begin{aligned}
\mathrm{P}_{1}= & \frac{\mu \mathrm{Q}^{2} w^{2}}{4 a v n^{4}}\left[\log \frac{1+n}{1-n}-\frac{2 n\left(3-\check{3} n^{2}\right)}{3\left(1-n^{2}\right)^{2}}\right. \\
& \left.-\sin ^{2} \psi\left\{\frac{3+n^{2}}{2} \log \frac{1+n}{1-n}-\frac{n\left(9-12 n^{2}-n^{4}\right)}{3\left(1-n^{2}\right)^{2}}\right\}\right] .
\end{aligned}
$$

If $\mathrm{O} 2$ (fig. 5) be in the plane of $u$ and $w$ and at right angles to $\mathbf{u}$, the cosine of the angle between $\mathrm{r}$ and $\mathrm{O}_{2}$ is $\sin \theta \cos \phi$. Hence, if $\mathrm{P}_{2}$ be the momentum in the direction of $\mathrm{O2}$,

$$
\mathbf{P}_{2}=\iint \frac{K E^{2}}{4 \pi v} 2 \alpha r^{2} \cos \phi \sin ^{2} \theta d \theta d \phi
$$

From (53) we find

$$
\begin{aligned}
& \int_{0}^{2 \pi} \cos \gamma \cos \phi d \phi=2 \pi \cdot \frac{1}{2} \sin \theta \sin \psi \\
& \int_{0}^{2 \pi} \cos ^{2} \gamma \cos \phi d \phi=2 \pi \cdot \sin \theta \cos \theta \sin \psi \cos \psi
\end{aligned}
$$

* Dr. O. Heaviside has calculated $\mathrm{W}$ for tinite values of $w$, when $\mathbf{w}$ is in the same direction as $\boldsymbol{u}$. 'Nature,' Nov, 6, 1902. 
Thus we obtain

$$
\mathrm{P}_{2}=\frac{\mu \mathrm{Q}^{2} w^{2} \sin \psi \cos \psi}{4 a v} \int_{0}^{\pi}\left\{\frac{n}{h^{3}}-\frac{\left(1-n^{2}\right) \cos \theta}{h^{4}}\right\} \sin ^{3} \theta d \theta
$$

Since $\cos \theta=(1-h) / n$ and $\sin \theta d \theta=d h / n$, this becomes

$$
\mathrm{P}_{2}=\frac{\mu \mathrm{Q}^{2} w^{2} \sin \psi \cos \psi}{4 a v n^{4}} \int_{i-n}^{1+n}\left\{\frac{\left(1-n^{2}\right)^{2}}{h^{4}}-\frac{3\left(1-n^{2}\right)}{h^{3}}+\frac{3-n^{2}}{h^{2}}-\frac{1}{h}\right\} d h .
$$

Hence, finally,

$$
\mathrm{P}_{2}=\frac{\mu \mathrm{Q}^{2} w^{2} \sin \psi \cos \psi}{4 a v n^{4}}\left[\frac{2 n\left(3-2 n^{2}\right)}{3\left(1-n^{2}\right)}-\log \frac{1+n}{1-n}\right]
$$

By symmetry the momentum in the pulse has no component perpendicular to the plane of $\mathbf{u}$ and $\mathbf{w}$.

$\S 33$. When $n$ is small but, of course, not zero we find that

$$
\mathrm{P}_{1}=\frac{w^{2} u m_{0}}{10 v^{2}}\left(4-\sin ^{2} \psi\right), \quad \mathrm{P}_{2}=\frac{v^{2} u m_{0}}{10 v^{2}} \sin \psi \cos \psi,
$$

where $m_{0}=2 \mu \mathrm{Q}^{2} / 3 a$, the electromagnetic mass of the sphere for infinitesimal speeds.

It is easily found that the whole momentum in the pulse can be expressed in the vector form

$$
\mathbf{P}=\left(m_{0} / 10 v^{2}\right)\left\{3 \mathbf{u} \cdot w^{2}+\mathbf{w}(\mathbf{u w})\right\} .
$$

The angle $\beta$ between $\mathbf{P}$ and $\boldsymbol{u}$ has its maximum value when $\tan \psi=2 / \sqrt{ } 3$, the maximum value being given by $\tan \beta=(48)^{-\frac{2}{2}}$. These values correspond to $\psi=49^{\circ} 6^{\prime}$ and $\beta=8^{\circ} 13^{\prime}$.

$\S 34$. When $n$ is nearly equal to unity, so that $\left(1-n^{2}\right)^{-1}$ is large, we again obtain simpie expressions for $\mathbf{P}_{1}$ and $\mathbf{P}_{2}$. Since $\log x$ is negligible in comparison with $x$ when $x$ is large, we see, by $\$ 32$, that, when $u$ is nearly equal to $v$,

$$
\mathrm{P}_{1}=\frac{m_{0} w^{2}\left(1-n^{2} \sin ^{2} \psi\right)}{2\left(1-n^{2}\right)^{2} v}, \quad \mathrm{P}_{2}=\frac{m_{0} w^{2} \sin \psi \cos \psi}{4\left(1-n^{2}\right) v}
$$

The resultant momentum in the pulse may be regarded as being in the direction of $\mathbf{u}$. For, if $\beta$ be the angle between $\mathbf{P}$ and $\boldsymbol{u}$, we have

$$
\tan \beta=\frac{\mathrm{P}_{2}}{\mathrm{P}_{1}}=\frac{\left(1-n^{2}\right) \sin \psi \cos \psi}{2\left(1-n^{2} \sin ^{2} \psi\right)},
$$

and $\tan \beta$ vanishes when $\psi=0$ and when $\psi=\frac{1}{2} \pi$. The maximum value of $\tan \beta$ is the small quantity $\frac{1}{2}\left(1-n^{2}\right)^{\frac{1}{2}}$, and this value occurs when $\tan \psi=\left(1-n^{2}\right)^{-\frac{1}{2}}$.

$\$ 35$. If the centre of the sphere be made to travel with velocity $u$ round a polygon inscribed in a circle of radius $r$, and if $2 \alpha$ be the angle which each side subtends at the centre, the vector change of velocity at each angle is L 2 
$w=2 u \sin \alpha$, and the angle between $w$ and $u$ is $\psi=\frac{1}{2} \pi-\alpha$. Then, by $\$ 31$, when $a$ is small, and therefore $w$ is small, the mean rate, $R$, of radiation of energy is

$$
\mathrm{R}=\frac{2 \mu \mathrm{Q}^{2} u^{3}\left(1-n^{2} \cos ^{2} \alpha\right) \sin \alpha}{3 a r\left(1-n^{2}\right)^{2}} .
$$

This result is applicable until $\alpha$ becomes so small that the pulse arising from one impulse hats not got clear of the sphere before the next impulse oceurs. The smallest permissible value of $\sin \alpha$ is $a u /(v-u) r$, and in this case

$$
\mathrm{R}=\frac{2 \mu \mathrm{Q}^{2} u^{2}}{3 v r^{2}\left(1-n^{2}\right)^{2}} \cdot \frac{1-n^{2} \cos ^{2} \alpha}{1-u}=\frac{1-n^{2} \cos ^{2} \alpha}{1-n} \mathrm{~K}_{0} .
$$

Here the radius of the sphere appears only through $\cos ^{2} \alpha$. If we make $a / r$ infinitesimal, we have $\cos \alpha=1$ and

$$
\mathrm{R}=(1+n) \mathrm{R}_{\mathrm{o}} \text {. }
$$

Dr. Heaviside* has shown that $R_{0}$ is the rate of radiation of energy from a true point-charge moving with velocity $\imath$ in a circle of radius $r$. Hence we see that, however small the sphere may be, the mean rate of radiation when the successive pulses just fail to overlap is greater than when they merge into a continuous disturbance.

$\$ 36$. If a point-charge $q$ has an acceleration $f$, the change of veloeity in time $d t$ is $\mathbf{f}$. $t$. The disturbance arising during this time is confined to a shell contained between two spheres, one of radius $v t$ described about the position of $q$ at the beginning of the interval, and the other of radius $v(t-d t)$ described about the position of $q$ at the end of the interval. The thickness of the shell is thus $(v-u \cos \theta) d t$ or $v h d t$ and the flux of displacement $\mathrm{K} e / 4 \pi$ is distributed through this thickness. Hence, putting $\mathrm{f} d t$ for $\mathrm{w}$ in $(50)$ and allowing for the thickness of the shell, we find that the electric force at a great distance from an accelerated point-charge is

$$
\mathbf{E}=\frac{q}{\mathrm{~K}} \cdot\left[-\frac{\mathbf{f}}{(u-u \cos \theta)^{2}}+\frac{\left(v \mathrm{r}_{1}-\mathrm{u}\right) f \cos \gamma}{(v-u \cos \theta)^{3}}\right]
$$

where $\theta$ and $\gamma$ are the angles made by $r$ with $u$ and $\mathbf{f}$. This result agrees with that given by $D_{r}$. Heaviside $\dagger$, who has also calculated the energy and the momentum carried away in the expanding shell.

$\$ 37$. In the preparation of this paper I have been greatly assisted by Mr. D. C. Jones of Pembroke College. He bais not only written out the manuscript for the press but has. also verified all the formula. My thanks are also due to Prof. J. J. Thomson for reading the paper in manuscript, and to Dr. A. H. Bucherer of Bonn for reading the proofs.

Cavendislı Laboratory, Cambridge.

* 'Nature,' Nov. 6, 1902, equation (10).

+ 'Nature, Nor. 6, 1002. See also M. Abraham, Theorie der Elektrizitit, ii. p. 97 . 